\title{
THE EQUIVALENCE OF CONTROLLED LAGRANGIAN AND CONTROLLED HAMILTONIAN SYSTEMS
}

\author{
Dong Eui Chang ${ }^{1}$, Anthony M. Bloch ${ }^{2}$, NaOmi E. Leonard ${ }^{3}$, \\ Jerrold E. Marsden ${ }^{1}$ And Craig A. Woolsey ${ }^{4}$
}

\begin{abstract}
The purpose of this paper is to show that the method of controlled Lagrangians and its Hamiltonian counterpart (based on the notion of passivity) are equivalent under rather general hypotheses. We study the particular case of simple mechanical control systems (where the underlying Lagrangian is kinetic minus potential energy) subject to controls and external forces in some detail. The equivalence makes use of almost Poisson structures (Poisson brackets that may fail to satisfy the Jacobi identity) on the Hamiltonian side, which is the Hamiltonian counterpart of a class of gyroscopic forces on the Lagrangian side.
\end{abstract}

Mathematics Subject Classification. 34D20, 70H03, 70H05, 93D15.

Received December 27, 2001. Revised February 26, 2002.

\section{INTRODUCTION}

Brief History and Background. Hamiltonian control systems were introduced by Brockett, Willems, and van der Schaft in [11,32-34,37]. They have been considerably developed since that time; see for example [16,30], and $[21,22]$, which extended the van der Schaft method to include systems with symmetry. One of the key methods introduced in these works is that of potential shaping to stabilize mechanical systems. Potential shaping refers to the use of feedback control to realize a modification to the potential energy of a given mechanical system, i.e., the control law is the force that derives from the potential energy modification. The controlled dynamics then resembles the original system dynamics, with a modification in the potential energy. The potential energy of the controlled system can be shaped for performance within the limitations of the available control authority. An important feature is that analysis tools for mechanical systems such as use of the energy as a Lyapunov function, can be applied to the controlled system since the closed-loop system dynamics describe a modified mechanical system.

Keywords and phrases: Controlled Lagrangian, controlled Hamiltonian, energy shaping, Lyapunov stability, passivity, equivalence.

1 Control and Dynamical Systems, California Institute of Technology, Pasadena, CA 91125, USA;

e-mail: dchang@cds.caltech.edu, marsden@cds.caltech.edu

Work partially supported by the California Institute of Technology and AFOSR grant ASOSR F49620-99-1-0190.

2 Department of Mathematics, University of Michigan, Ann Arbor, MI 48109, USA; e-mail: abloch@math.1sa.umich.edu

Work partially supported by NSF grants DMS 981283 and 0103895 and AFOSR.

3 Mechanical \& Aerospace Engineering, Princeton University, Princeton, NJ 08544, USA; e-mail: naomi@princeton.edu

Work partially supported by NSF grant CCR-9980058, ONR grant N00014-98-1-0649 and AFOSR grant F49620-01-1-0382.

4 Aerospace \& Ocean Engineering, Virginia Tech., Blacksburg, VA 24061, USA; e-mail: cwoolsey@vt.edu 
The idea behind the method of controlled Lagrangians was introduced by Krishnaprasad, Bloch, Marsden and Sanchez, starting with [25], and [7]. Interestingly [7] emphasized the Hamiltonian and non-canonical Poisson bracket approach. This was put into a systematic Lagrangian context in a series of papers of Bloch, Leonard and Marsden, starting with [8]. These works introduced the important notion of kinetic shaping which is analogous to potential shaping except that the control law is selected to realize a desired modification to kinetic energy, i.e., the control law is the force that derives from the kinetic energy modification. In this case the controlled system dynamics resemble the original dynamics but with a modified kinetic energy. In these early papers it is shown how kinetic shaping can be applied to underactuated systems (such as a spacecraft stabilized by an internal rotor and the inverted pendulum on a cart), for which potential shaping methods are not applicable. Since that time, this approach has matured and developed considerably and has been applied to many systems, such as spherical pendula and underwater vehicles, as in for example [3,9] and references therein. The addition of damping was studied in $[39,40]$ and some preliminary tracking results were given in [3]. We shall refer to this class of systems as controlled Lagrangian systems, or CL systems for short.

In tandem with the development of energy shaping methodology, possibilities for structure modification have been investigated. For example, in the papers [25] and [7], the authors demonstrated, in the context of the spacecraft dynamics problem, the concept of using feedback control to modify the system's Poisson structure. Structure-modifying control laws were designed to stabilize an underwater vehicle with internal rotors in the works $[38,41]$ of Woolsey and Leonard. In the underwater vehicle work, the modified structure is almost LiePoisson, i.e., the Poisson structure for the controlled system does not satisfy the Jacobi identity. The idea of structure modification is somewhat analogous and certainly complementary to the notion of energy shaping. In structure modification, the closed-loop system is a mechanical system but the structure (e.g., the Poisson structure) is different from the structure of the original dynamics. Modifying both structure and energy by means of feedback control gives the opportunity for more flexibility (i.e., a larger class of control inputs is considered) as compared to energy shaping alone, while the advantages of providing a closed-loop system that is equivalent to a mechanical system are retained. Recent developments on controlled Lagrangian systems with modified structure were presented in [4].

For purposes of the present work, two important recent references on the Hamiltonian side are those of Ortega, Spong, Gómez-Estern, Blankenstein and van der Schaft [29] and [2]. We shall refer to the Hamiltonian versions of systems introduced and studied in these papers as controlled Hamiltonian systems or CH systems for short. The paper [29] develops a formulation of passivity-based control using interconnection and damping assignment, or IDA-PBC. See [28] and [35] for additional applications of the passivity-based control approach. The idea, as in the controlled Lagrangian approach, is that it applies to the problem of stabilizing underactuated mechanical systems using both potential and kinetic shaping. In [29], the authors also discuss structure modification, including modifications of the Poisson structure.

Purpose of this Paper. The main purpose of this paper is to show that the CL system method is equivalent to the CH system method; we shall focus on simple mechanical control systems (where the Lagrangian has the form of kinetic minus potential energy), although this is true more generally, as will be evident in the body of the paper. One of the interesting features is that we identify the Lagrangian analog of the failure of Jacobi's identity on the Hamiltonian side, namely in terms of gyroscopic forces, which is a natural Lagrangian concept. This then provides a framework on the Lagrangian as well as the Hamiltonian side for considering the problem of general structure modification.

In addition to showing this equivalence, in a companion paper we shall extend both the Lagrangian and Hamiltonian sides of this theory to include systems with symmetry and the relevant reduction theory (Lagrangian reduction, where one reduces variational principles and Hamiltonian reduction, where one reduces symplectic and Poisson structures). This extension is critical for examples like spacecraft control, underwater vehicle control, etc. In fact, this class of reduced systems was recognized early as an important one on both the Hamiltonian and Lagrangian sides; see [25] and [7]. 
Notation. We shall use fairly standard notation, as follows: the configuration manifold for the mechanical system under consideration will be denoted $Q$, the Lagrangian will be denoted $L: T Q \rightarrow \mathbb{R}$ and Hamiltonians will be denoted $H: T^{*} Q \rightarrow \mathbb{R}$. We will assume that $Q$ is finite dimensional and denote the dimension by $n$. The second order tangent bundle is denoted $T^{(2)} Q$ and consists of second derivatives of curves in $Q$. See [26] and [13] for additional details. Coordinates on $Q$ will be denoted $\left(q^{1}, \ldots, q^{n}\right)$. Likewise, coordinates on $T Q$ are denoted $\left(q^{1}, \ldots, q^{n}, \dot{q}^{1}, \ldots \dot{q}^{n}\right)$ and coordinates on $T^{*} Q$ are denoted $\left(q^{1}, \ldots, q^{n}, p_{1}, \ldots, p_{n}\right)$.

Strategy. The procedure introduced in [8] (and used in the more recent works of [9, 10] and [3] as well), can be described in general terms, as follows. One starts with an underactuated $n$-degree of freedom mechanical control system of the form

$$
\begin{aligned}
& \frac{\mathrm{d}}{\mathrm{d} t} \frac{\partial L}{\partial \dot{q}^{i}}-\frac{\partial L}{\partial q^{i}}=u_{i}, \quad i=1, \ldots, k \\
& \frac{\mathrm{d}}{\mathrm{d} t} \frac{\partial L}{\partial \dot{q}^{i}}-\frac{\partial L}{\partial q^{i}}=0, \quad i=k+1, \ldots, n .
\end{aligned}
$$

Thus, the degrees of freedom represented by $q^{1}, \ldots, q^{k}$ are the actuated degrees of freedom and the remaining are unactuated. One then seeks a new controlled Lagrangian $L_{c}$ and a class of feedback controls $u_{1}, \ldots, u_{k}$ such that the system $(1,2)$ is equivalent to the Euler-Lagrange equations for $L_{c}$ with control $u_{c}$ of rank $k$ :

$$
\frac{\mathrm{d}}{\mathrm{d} t} \frac{\partial L_{c}}{\partial \dot{q}}-\frac{\partial L_{c}}{\partial q}=u_{c} .
$$

The requirement that these two systems be equivalent leads to the problem of matching for which many specific techniques, including potential and kinetic shaping were developed. Some important general matching techniques were developed by Auckly et al. in [1] and by Hamberg in [18, 19]; these were important inspirations for the approach in this paper.

The matching process usually has some free parameters which can be interpreted as control gains. The advantage of the form (3) is explained as follows: one chooses a set of appropriate parameters such that the energy $E_{c}$ corresponding to the Lagrangian $L_{c}$ has a minimum at the equilibrium of interest. The control $u_{c}$ is chosen to be a control that is a dissipative, possibly together with a gyroscopic, force. One then uses the energy $E_{c}$ as a Lyapunov function to which LaSalle's invariance principle can be applied to demonstrate stabilization. One often has a demonstrably large region of attraction.

In the present paper, we view this matching problem in a different way that turns out to be quite crucial. We consider the problem of determining the equivalence of two possibly underactuated and/or externally forced CL systems. Then the matching problem becomes one of determining when $(1,2)$ and $(3)$ are CL-equivalent. We shall also develop the notion of $\mathrm{CH}$-equivalent systems and likewise use this to deal with the matching problem on the Hamiltonian side. While this may seem at this point to be a rather superficial reformulation of the problem, as we shall see, it is a powerful point of view that enables us to elegantly achieve our goals.

There is another important point here; in our previous papers (see $[3,9,10]$ ), we developed methods that make control design systematic and constructive for certain classes of mechanical systems, which avoided having to deal with solving PDE's for each new application. In this context, these papers also dealt with the important issues of asymptotic stabilization and gave an indication that the methods are also useful for tracking. These are obviously important points from the view of applications. However, fully exploiting and generalizing all of these results is not the purpose of the present paper; rather it is to develop the general notion of equivalence and to show that the CL method is equivalent to the $\mathrm{CH}$ method. We shall, however, see how the constructive methods mentioned can be derived in the general context of the present work.

Motivating Example. Consider a model of an inverted pendulum on a cart, an example we will return to in the next section (see Fig. 1). We use it now to motivate the discussion and illustrate some of the ideas. 


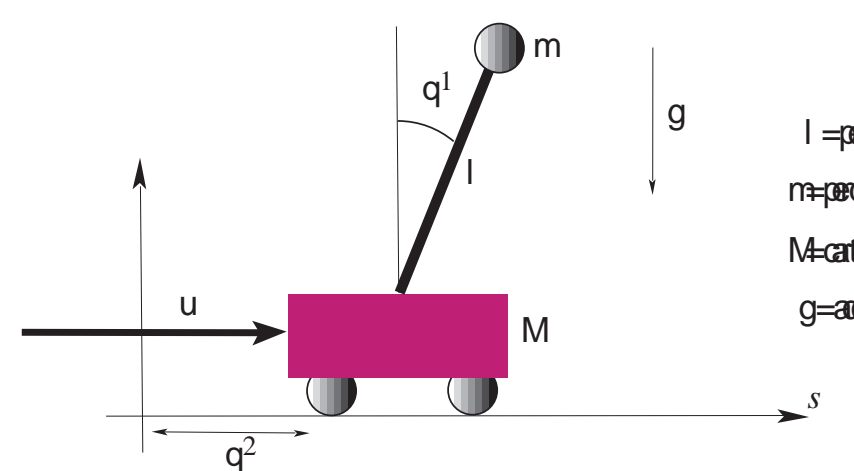

Figure 1. The pendulum on a cart.

The Lagrangian $L$ of the system is given by

$$
L\left(q^{1}, q^{2}, \dot{q}^{2}, \dot{q}^{2}\right)=\frac{1}{2}\left(\dot{q}^{1}\right)^{2}+\cos \left(q^{1}\right) \dot{q}^{1} \dot{q}^{2}+\left(\dot{q}^{2}\right)^{2}-\cos \left(q^{1}\right),
$$

where $q^{1}$ is the angle of the pendulum from the vertical position and $q^{2}$ is the displacement of the cart from the origin. Here, all the parameter values (i.e., those identified in Fig. 1) are taken to have unit value. The control $u$ is exerted along the $q^{2}$ variable as shown in the figure. The equations of motion with control $u$ is given by

$$
\ddot{q}^{1}+\cos \left(q^{1}\right) \ddot{q}^{2}-\sin \left(q^{1}\right)=0, \quad \cos \left(q^{1}\right) \ddot{q}^{1}+2 \ddot{q}^{2}-\sin \left(q^{1}\right)\left(\dot{q}^{1}\right)^{2}=u
$$

The goal is to design a feedback control law $u$ such that the point $\left(q^{1}, q^{2}, \dot{q}^{1}, \dot{q}^{2}\right)=(0,0,0,0)$ becomes an asymptotically stable equilibrium for the dynamics (5). The energy $E$ of the Lagrangian $L$ is given by

$$
E\left(q^{1}, q^{2}, \dot{q}^{1}, \dot{q}^{2}\right)=\frac{1}{2}\left(\dot{q}^{1}\right)^{2}+\cos \left(q^{1}\right) \dot{q}^{1} \dot{q}^{2}+\left(\dot{q}^{2}\right)^{2}+\cos \left(q^{1}\right) .
$$

One cannot directly use the energy $E$ as a Lyapunov function because $(0,0,0,0)$ is a saddle point of $E$.

The method of controlled Lagrangians leads one to the following (non-obvious and, at the moment, mysterious) form of control $u$ :

$$
u=\frac{199\left(\left(\dot{q}^{1}\right)^{2}-\cos \left(q^{1}\right)\right) \sin \left(q^{1}\right)+\left(2-\cos ^{2}\left(q^{1}\right)\right)\left(v-\frac{1}{500} \sin \left(q^{1}\right)-\frac{1}{100000} q^{2}\right)}{1-100 \cos ^{2}\left(q^{1}\right)}
$$

where $v$ is to be chosen. In (6), the somewhat strange numerical parameters were chosen to get a large region of attraction, which will be discussed later. Then, one can show either by a lengthy direct calculation, or by use of the theory, that the dynamics of the closed-loop system $(5)+(6)$, is the same as the dynamics of the Lagrangian

$$
L_{c}=\frac{20000 \cos ^{2}\left(q^{1}\right)-100}{2}\left(\dot{q}^{1}\right)^{2}+100 \cos \left(q^{1}\right) \dot{q}^{1} \dot{q}^{2}+\frac{1}{2} \dot{q}^{2}+100 \cos \left(q^{1}\right)-\frac{1}{200000}\left(q^{2}+200 \sin \left(q^{1}\right)\right)^{2}
$$

with the control force

$$
u_{c}=v\left[\begin{array}{c}
200 \cos \left(q^{1}\right) \\
1
\end{array}\right]
$$


where the parameter value $1 / 200000$ in the potential energy of $L_{c}$ corresponds to a slow driving of the cart to the origin as compared to a rapid stabilization of the pendulum to the upright position. The energy $E_{c}$ of the Lagrangian $L_{c}$ is given by

$$
E_{c}=\frac{20000 \cos ^{2}\left(q^{1}\right)-100}{2}\left(\dot{q}^{1}\right)^{2}+100 \cos \left(q^{1}\right) \dot{q}^{1} \dot{q}^{2}+\frac{1}{2} \dot{q}^{2}-100 \cos \left(q^{1}\right)+\frac{1}{200000}\left(q^{2}+200 \sin \left(q^{1}\right)\right)^{2}
$$

and has a minimum at $(0,0,0,0)$ in the set

$$
S:=\left\{\left(q^{1}, q^{2}, \dot{q}^{1}, \dot{q}^{2}\right) \mid 100 \cos ^{2}\left(q^{1}\right)>1\right\}=\left(-0.9365 \times \frac{\pi}{2}, 0.9365 \times \frac{\pi}{2}\right) \times \mathbb{R}^{3} .
$$

The minimum value of $E_{c}$ is $(-100)$, and the kinetic energy of $E_{c}$ is positive definite in the set $S$. We are now in a position to use $E_{c}$ as a Lyapunov function for the dynamics of the system $\left(L_{c}, u_{c}\right)$, (or, equivalently, $(L, u)$ ). The time derivative of $E_{c}$ is given by

$$
\frac{\mathrm{d} E_{c}}{\mathrm{~d} t}=\left\langle\left(\dot{q}^{1}, \dot{q}^{2}\right), u_{c}\right\rangle=v\left(200 \dot{q}^{1} \cos \left(q^{1}\right)+\dot{q}^{2}\right) .
$$

We choose $v=-\left(200 \dot{q}^{1} \cos \left(q^{1}\right)+\dot{q}^{2}\right)$ so that $u_{c}$ becomes dissipative, i.e.,

$$
\frac{\mathrm{d} E_{c}}{\mathrm{~d} t}=-\left(200 \dot{q}^{1} \cos \left(q^{1}\right)+\dot{q}^{2}\right)^{2} \leq 0
$$

Hence $(0,0,0,0)$ becomes a Lyapunov stable equilibrium of the closed-loop system. By applying LaSalle's theorem (see, for example [23]), one can also show asymptotic stability of $(0,0,0,0)$ in a region of attraction, $R:=E_{c}^{-1}([-100,-11]) \bigcap S$. Notice that $R$ is a large region of attraction because such points as $(0,4000,0,0)$ and $\left(0.908 \times \frac{\pi}{2}, 0,0,0\right)$ are contained in it. But it is still a conservative choice of a region of attraction and by adjusting the parameters one can find even larger region of an attraction.

Recap of the Discussion. First, we find a Lagrangian $L_{c}$ and a control $u_{c}$ so that the Lagrangian control system $\left(L_{c}, u_{c}\right)$ is equivalent to the original system, $(L, u)$. The energy $E_{c}$ of $L_{c}$ has a minimum at $(0,0,0,0)$, whereas the energy $E$ of the original $L$ does not. Hence, we are able to use $E_{c}$ for a Lyapunov function and, to achieve asymptotic stability of $(0,0,0,0)$, we choose dissipation for $u_{c}$. A large region of attraction is achievable.

Clearly, this technique depends on a good theory for the equivalence of two Euler-Lagrange systems with control forces. In this paper we provide such a theory; in particular, we show how to find systems equivalent to a given system. We also go beyond this to our second main point, namely a proof of the equivalence of the CL point of view and the $\mathrm{CH}$ point of view.

\section{Controlled Lagrangian systems}

To formally define a controlled Lagrangian system, we need to first recall a few things about the EulerLagrange equations from basic geometric mechanics (see, for example [26]).

Euler-Lagrange Equations. The Euler-Lagrange operator $\mathcal{E} \mathcal{L}$ assigns to a Lagrangian $L: T Q \rightarrow \mathbb{R}$, a bundle map $\mathcal{E} \mathcal{L}(L): T^{(2)} Q \rightarrow T^{*} Q$ which may be written in local coordinates (and with the summation convention in force) as

$$
\mathcal{E} \mathcal{L}(L)_{i}(q, \dot{q}, \ddot{q}) \mathrm{d} q^{i}=\left(\frac{\mathrm{d}}{\mathrm{d} t} \frac{\partial L}{\partial \dot{q}^{i}}(q, \dot{q})-\frac{\partial L}{\partial q^{i}}(q, \dot{q})\right) \mathrm{d} q^{i}
$$

in which it is understood that one regards the first term on the right hand side as a function on the second order tangent bundle $T^{(2)} Q$ by formally applying the chain rule and then replacing everywhere $\mathrm{d} q / \mathrm{d} t$ by $\dot{q}$ and 
$\mathrm{d} \dot{q} / \mathrm{d} t$ by $\ddot{q}$. Hence the Euler-Lagrange equations of a Lagrangian $L$ may be written as

$$
\mathcal{E} \mathcal{L}(L)(q, \dot{q}, \ddot{q})=0
$$

Controlled Lagrangian Systems. The view of a controlled Lagrangian system given by $(1,2)$ will now be generalized to include external forces and also will be made intrinsic (independent of a specific coordinate representation). This definition is fundamental to the Lagrangian side of this work.

Definition 2.1. A controlled Lagrangian (CL) system is a triple $(L, F, W)$ where the function $L: T Q \rightarrow \mathbb{R}$ is the Lagrangian, the fiber-preserving map $F: T Q \rightarrow T^{*} Q$ is an external force and $W \subset T^{*} Q$ is a subbundle of $T^{*} Q$, called the control bundle, representing the actuation directions.

Sometimes, we will identify the subbundle $W$ with the set of bundle maps from $T Q$ to $W$. The fact that $W$ may be smaller than the whole space corresponds to the system being underactuated. The equations of motion of the system $(L, F, W)$ may be written as

$$
\mathcal{E} \mathcal{L}(L)(q, \dot{q}, \ddot{q})=F(q, \dot{q})+u
$$

with a control $u$ selected from $W$. When we choose a specific control map $u: T Q \rightarrow W$ (so that $u$ is a function of $\left.\left(q^{i}, \dot{q}^{i}\right)\right)$, then we call the triple $(L, F, u)$ a closed-loop Lagrangian system. We will typically be interested in such feedback controls in this paper.

In the special case when $W$ is integrable (that is, its annihilator $W^{\circ} \subset T Q$ is integrable in the usual Frobenius sense) and we choose coordinates appropriately, then a CL system, that is the equations (10), can be locally written in coordinates as

$$
\begin{array}{ll}
\frac{\mathrm{d}}{\mathrm{d} t} \frac{\partial L}{\partial \dot{q}^{i}}-\frac{\partial L}{\partial q^{i}}=F_{i}+u_{i}, & i=1, \ldots, k \\
\frac{\mathrm{d}}{\mathrm{d} t} \frac{\partial L}{\partial \dot{q}^{i}}-\frac{\partial L}{\partial q^{i}}=F_{i}, & i=k+1, \ldots, n .
\end{array}
$$

Here the coordinates $q^{1}, \ldots, q^{k}$ are chosen so that $\mathrm{d} q^{1}, \ldots \mathrm{d} q^{k}$ span $W$, so $W$ is $k$ dimensional in this case. The external forces can include gyroscopic forces, friction forces, etc.

Aside: Interconnection Structures and Graphs. If one has a collection of CL systems $\left(L^{i}, F^{i}, W^{i}\right)$, $i=1, \ldots, N$ (as occurs in the coordinated control of a collection of $N$ satellites or $N$ underwater vehicles, for example), then one can keep track of the interconnection structure by using a directed graph with the individual CL system representing the nodes of the graph and the interconnection structure representing the edges. If, for example, in this graph, there is a directed link from the $i$ th subsystem $\left(L^{i}, F^{i}, W^{i}\right)$ to the $j$ th subsystem $\left(L^{j}, F^{j}, W^{j}\right)$, then we would have a control link $u: T Q_{i} \rightarrow W_{j}$. We intend to pursue this point of view in future publications for purposes of coordinated control of many subsystems. For simplicity of the exposition in the present paper, we assume that we have lumped everything together into one system.

Simple CL Systems. Now we are ready to embark on the discussion of the matching problem in the general context of CL systems. We shall begin with the case of simple Lagrangians. 
Definition 2.2. A CL system $(L, F, W)$ is called a simple $C \boldsymbol{L}$ system if the Lagrangian $L$ has the form of kinetic minus potential energy:

$$
L(q, \dot{q})=\frac{1}{2} m(q)(\dot{q}, \dot{q})-V(q)
$$

where $m$ is a non-degenerate symmetric $(0,2)$-tensor (the mass matrix, or Riemannian structure).

We will sometimes omit the $q$-dependence of $m$ in the notation, but this dependence will be understood. When $L$ is a simple Lagrangian, then the Euler-Lagrange operator is written in a matrix form as

$$
\mathcal{E} \mathcal{L}(L)(q, \dot{q}, \ddot{q})=m \ddot{q}+(\mathbf{d} m[\dot{q}]) \dot{q}-\frac{\partial L}{\partial q}
$$

where $(\mathbf{d} m[\dot{q}])_{i j}=\mathbf{d}\left(m_{i j}\right)(\dot{q})$, or in coordinates,

$$
\mathcal{E} \mathcal{L}(L)(q, \dot{q}, \ddot{q})_{j}=m_{i j} \ddot{q}^{i}+\frac{\partial m_{i j}}{\partial q^{k}} \dot{q}^{i} \dot{q}^{k}-\frac{1}{2} \frac{\partial m_{i k}}{\partial q^{j}} \dot{q}^{i} \dot{q}^{k}+\frac{\partial V}{\partial q^{j}} .
$$

See, for example, reference [26] for how to relate these expressions to Christoffel symbols and geodesics. We make this assumption of a simple CL system only for the sake of simplicity and to make the exposition more concrete. One can readily generalize the results to more general forms of Lagrangians (see Rem. 1 below).

Notation. As is standard, we represent the two tensor $m$ in coordinates with indices down: $m_{i j}$. This corresponds to the fact that at each point $q \in Q, m$ is a bilinear map $m_{q}: T_{q} Q \times T_{q} Q \rightarrow \mathbb{R}$. If $m$ is non-degenerate, it defines an isomorphism $m_{q}^{b}: T_{q} Q \rightarrow T_{q}^{*} Q$ by $m_{q}^{b}\left(v_{q}\right) \cdot u_{q}=m_{q}\left(v_{q}, u_{q}\right)$. The matrix representation of $m$ and $m^{b}$ are the same and correspond to simply writing the mass matrix as an $n \times n$ matrix. The inverse of the linear map $m_{q}^{b}$ is denoted $m_{q}^{-1}: T_{q}^{*} Q \rightarrow T_{q} Q$. Its matrix is denoted $m^{i j}$; it is the inverse of the standard mass matrix and satisfies $m_{i j} m^{j k}=\delta_{i}^{k}$. To not complicate the notation, we will let $m$ denote both the bilinear form on $T_{q} Q$ and the associated linear map of $T_{q} Q$ to $T_{q}^{*} Q$.

The Matching Conditions and CL-Equivalence. Consider now two simple Lagrangian systems $\left(L_{1}, F_{1}, W_{1}\right)$ and $\left(L_{2}, F_{2}, W_{2}\right)$ with

$$
L_{1}(q, \dot{q})=\frac{1}{2} m_{1}(\dot{q}, \dot{q})-V_{1}(q) \quad \text { and } \quad L_{2}(q, \dot{q})=\frac{1}{2} m_{2}(\dot{q}, \dot{q})-V_{2}(q) .
$$

The $\ddot{q}$ equation of the closed-loop system $\left(L_{\alpha}, F_{\alpha}, u_{\alpha}\right)$ with $\alpha=1,2$ is given in coordinates as follows:

$$
\ddot{q}_{L_{\alpha}}=m_{\alpha}^{-1}\left[-\left(\mathbf{d} m_{\alpha}[\dot{q}]\right) \dot{q}+\frac{\partial L_{\alpha}}{\partial q}+F_{\alpha}+u_{\alpha}\right] .
$$

Here we use matrix and vector-style notation, suppressing the indices on $q^{i}$ to avoid cluttering the equation with too many indices. We can then formally define matching conditions between these two systems.

Definition 2.3. Given the two CL systems $\left(L_{1}, F_{1}, W_{1}\right)$ and $\left(L_{2}, F_{2}, W_{2}\right)$, the Euler-Lagrange matching conditions are

ELM-1: $W_{1}=m_{1} m_{2}^{-1}\left(W_{2}\right)$,

ELM-2: $\operatorname{Im}\left[\left(\mathcal{E} \mathcal{L}\left(L_{1}\right)-F_{1}\right)-m_{1} m_{2}^{-1}\left(\mathcal{E} \mathcal{L}\left(L_{2}\right)-F_{2}\right)\right] \subset W_{1}$,

where Im means the pointwise image of the map in brackets.

We say that the two simple CL Lagrangian systems $\left(L_{1}, F_{1}, W_{1}\right)$ and $\left(L_{2}, F_{2}, W_{2}\right)$ are CL-equivalent if

ELM-1 and ELM-2 hold. We use the symbol, $\stackrel{L}{\sim}$ for this equivalence relation. 
One can show that CL-equivalence is indeed an equivalence relation. The reflexivity and the transitivity are obvious. The symmetry follows if we multiply both sides of ELM-1 and ELM-2 by $m_{2} m_{1}^{-1}$.

In coordinates and in terms of concrete mass matrices, the condition ELM-1 means that the matrix product $m_{1} m_{2}^{-1}$ takes the subspace associated to the actuation directions of the second system to the subspace associated to the actuation directions of the first system; this may of course be viewed as a linear algebra condition. The condition ELM-2 means that the given combination of Euler-Lagrange expressions and forces lies in the actuation direction of the first system. If $\left(L_{1}, F_{1}, W_{1}\right)$ and $\left(L_{2}, F_{2}, W_{2}\right)$ are equivalent, then for any choice of control $u_{2}: T Q \rightarrow W_{2}$, there is a control $u_{1}: T Q \rightarrow W_{1}$ such that such that the two closed-loop systems $\left(L_{1}, F_{1}, u_{1}\right)$ and $\left(L_{2}, F_{2}, u_{2}\right)$ produce the same equations of motion, and vice versa. The relation between such $u_{1}$ and $u_{2}$ is given by (17).

One can easily check by coordinate computation that the map

$$
\left[\left(\mathcal{E} \mathcal{L}\left(L_{1}\right)-F_{1}\right)-m_{1} m_{2}^{-1}\left(\mathcal{E} \mathcal{L}\left(L_{2}\right)-F_{2}\right)\right]
$$

in ELM-2 can be regarded as a map defined on $T Q$ because the acceleration terms from the two Euler-Lagrange expressions cancel each other.

Equations of Motion. Now we can give the main result on when two CL systems give the same equations of motion.

Proposition 2.4. Suppose two simple controlled Lagrangian systems $\left(L_{1}, F_{1}, W_{1}\right)$ and $\left(L_{2}, F_{2}, W_{2}\right)$ with Lagrangians in (14) are CL-equivalent. Then the two closed-loop systems $\left(L_{1}, F_{1}, u_{1}\right)$ and $\left(L_{2}, F_{2}, u_{2}\right)$ produce the same equations of motion if and only if the two feedback controls $u_{1}: T Q \rightarrow W_{1}$ and $u_{2}: T Q \rightarrow W_{2}$ satisfy

$$
u_{1}=\left(\mathcal{E} \mathcal{L}\left(L_{1}\right)-F_{1}\right)-m_{1} m_{2}^{-1}\left(\mathcal{E} \mathcal{L}\left(L_{2}\right)-F_{2}\right)+m_{1} m_{2}^{-1} u_{2}
$$

Proof. By (15) and (13) one can compute

$$
m_{1}\left(\ddot{q}_{L_{1}}-\ddot{q}_{L_{2}}\right)=u_{1}-m_{1} m_{2}^{-1} u_{2}-\left[\left(\mathcal{E} \mathcal{L}\left(L_{1}\right)-F_{1}\right)-m_{1} m_{2}^{-1}\left(\mathcal{E} \mathcal{L}\left(L_{2}\right)-F_{2}\right)\right] .
$$

The conditions ELM-1 and ELM-2 imply that (17) holds if and only if $\ddot{q}_{1}=\ddot{q}_{2}$. Also recall that the map in (16) can be regarded as a map defined on $T Q$.

Coordinate Expressions. We express the Euler-Lagrange matching conditions in coordinates. Let $\left(L_{1}, F_{1}, W_{1}\right)$ and $\left(L_{2}, F_{2}, W_{2}\right)$ be two simple CL systems with Lagrangians as in (14). Suppose that we are given a decomposition of the forces $F_{i}$ as

$$
F_{i}=F_{i}^{v}+F_{i}^{q}
$$

with $i=1,2$ where $F_{i}^{q}$ is independent of the velocity $\dot{q}$. Then ELM-2 can be written in coordinates as

$$
\begin{aligned}
W_{1}^{\circ}\left[\left(\mathbf{d} m_{1}[\dot{q}]\right) \dot{q}\right. & -\frac{\partial}{\partial q}\left(\frac{1}{2} \dot{q}^{T} m_{1} \dot{q}\right)-F_{1}^{v}(q, \dot{q}) \\
& \left.-m_{1} m_{2}^{-1}\left(\left(\mathbf{d} m_{2}[\dot{q}]\right) \dot{q}-\frac{\partial}{\partial q}\left(\frac{1}{2} \dot{q}^{T} m_{2} \dot{q}\right)-F_{2}^{v}(q, \dot{q})\right)\right]=0
\end{aligned}
$$

and

$$
W_{1}^{\circ}\left[\frac{\partial V_{1}}{\partial q}-F_{1}^{q}-m_{1} m_{2}^{-1}\left(\frac{\partial V_{2}}{\partial q}-F_{2}^{q}\right)\right]=0
$$


where $W_{1}^{\circ}$ is a matrix whose rows span the annihilator of $W_{1}$, given by

$$
\left\{v \in T Q \mid\langle v, \alpha\rangle=0 \text { for all } \alpha \in W_{1}\right\}
$$

CL-Inclusion. There is a more general concept than the CL-equivalence relation. We will give this definition for simple Lagrangian systems, but it can be readily generalized for general Lagrangian systems (see Rem. 1 below).

Definition 2.5. We say that a simple CL system $\left(L_{1}, F_{1}, W_{1}\right)$ includes the simple CL system $\left(L_{2}, F_{2}, W_{2}\right)$ if the following holds:

ELI-1: $W_{1} \supset m_{1} m_{2}^{-1}\left(W_{2}\right)$,

ELI-2: $\operatorname{Im}\left[\left(\mathcal{E} \mathcal{L}\left(L_{1}\right)-F_{1}\right)-m_{1} m_{2}^{-1}\left(\mathcal{E} \mathcal{L}\left(L_{2}\right)-F_{2}\right)\right] \subset W_{1}$.

If $\left(L_{1}, F_{1}, W_{1}\right)$ includes $\left(L_{2}, F_{2}, W_{2}\right)$, then for any choice of control $u_{2}: T Q \rightarrow W_{2}$, there is a control $u_{1}$ : $T Q \rightarrow W_{1}$ satisfying (17) such that the two closed-loop systems $\left(L_{1}, F_{1}, u_{1}\right)$ and $\left(L_{2}, F_{2}, u_{2}\right)$ produce the same equations of motion. This introduces a partial order in the class of simple controlled Lagrangian systems. We call this partial order $\boldsymbol{C L}$-inclusion. One can check that two simple CL systems include each other if and only if they are $C L$-equivalent.

Remarks. 1. In this paper we always assume that Lagrangians are regular, i.e., $\operatorname{det}\left(\frac{\partial^{2} L}{\partial \dot{q}^{i} \partial \dot{q}^{j}}\right) \neq 0$. For simple CL systems this means of course that the mass matrix is nonsingular. We can generalize the Euler-Lagrange matching conditions for general Lagrangians which are not necessarily simple as follows. Let $L: T Q \rightarrow \mathbb{R}$ be a Lagrangian. It induces a globally well-defined map $m_{L}: T Q \rightarrow \operatorname{Sym}_{2}\left(T^{*} Q\right.$ ) (symmetric two-tensors with indices down) given in tangent bundle charts as follows:

$$
m_{L}(q, \dot{q})=\frac{\partial^{2} L(q, \dot{q})}{\partial \dot{q}^{i} \partial \dot{q}^{j}} \mathrm{~d} q^{i} \otimes \mathrm{d} q^{j} .
$$

Then the Euler-Lagrange matching conditions for general Lagrangian systems $\left(L_{1}, F_{1}, W_{1}\right)$ and $\left(L_{2}, F_{2}, W_{2}\right)$ are given by

ELM-1': $W_{1}=m_{L_{1}} m_{L_{2}}^{-1}\left(W_{2}\right)$,

ELM-2': $\operatorname{Im}\left[\left(\mathcal{E} \mathcal{L}\left(L_{1}\right)-F_{1}\right)-m_{L_{1}} m_{L_{2}}^{-1}\left(\mathcal{E} \mathcal{L}\left(L_{2}\right)-F_{2}\right)\right] \subset W_{1}$.

The equation in (17) is replaced by

$$
u_{1}=\left(\mathcal{E} \mathcal{L}\left(L_{1}\right)-F_{1}\right)-m_{L_{1}} m_{L_{2}}^{-1}\left(\mathcal{E} \mathcal{L}\left(L_{2}\right)-F_{2}\right)-m_{L_{1}} m_{L_{2}}^{-1} u_{2}
$$

so that the two closed-loop systems $\left(L_{1}, F_{1}, u_{1}\right)$ and $\left(L_{2}, F_{2}, u_{2}\right)$ produce the same equations of motion.

2. One can approach the Euler-Lagrange equations using the Lagrangian two-form (see Sect. 7.3 of [26] for details). However, in this paper, we do not take this approach but rather take the variational approach since the variational approach is more natural for many purposes, including Lagrangian reduction; we will use this variational to discuss reduced CL and $\mathrm{CH}$ systems in a companion paper (see [13] for an overview of the theory of Lagrangian reduction). Since Section 7.3 of [26] does not consider external forces, we give a formula for the Lagrangian vector field $X_{E}$ for a given Lagrangian system $(L, F, W)$ with the energy function $E$ defined by

$$
E(q)=\langle\mathbb{F} L(q, \dot{q}),(q, \dot{q})\rangle-L(q, \dot{q})
$$

We recall that the Legendre transformation $\mathbb{F} L$ is defined as follows: for $v_{q}, w_{q} \in T_{q} Q$

$$
\left\langle\mathbb{F} L\left(v_{q}\right), w_{q}\right\rangle=\left.\frac{\mathrm{d}}{\mathrm{d} s}\right|_{s=0} L\left(v_{q}+s w_{q}\right) .
$$


In coordinates,

$$
\left(q^{i}, p_{i}\right)=\mathbb{F} L(q, \dot{q})=\left(q^{i}, \frac{\partial L}{\partial \dot{q}^{i}}\right)
$$

If $L(q, \dot{q})=\frac{1}{2} m(\dot{q}, \dot{q})-V(q)$ is simple, then

$$
\mathbb{F} L=m: T Q \rightarrow T^{*} Q
$$

is a linear isomorphism on each fiber.

The Lagrangian vector field $X_{E}$ on $T Q$ of the system $(L, F, W)$ and with with controls $u: T Q \rightarrow W$ is verified (using computations similar to those in, for example [26]), to be given by

$$
X_{E}=\Omega_{L}^{\sharp} \mathbf{d} E+T \mathbb{F} L^{-1}\left(\operatorname{vlift}_{m}(F)\right)+T \mathbb{F} L^{-1}\left(\operatorname{vlift}_{m}(u)\right) .
$$

The notation in this equation is as follows: $T \mathbb{F} L^{-1}: T\left(T^{*} Q\right) \rightarrow T(T Q)$ is the tangent map of the inverse Legendre transformation $\mathbb{F} L^{-1}: T^{*} Q \rightarrow T Q$, and $\Omega_{L}$ is the Lagrangian two form on $T Q$ defined by $\Omega_{L}=$ $\mathbb{F} L^{*} \Omega_{\text {can }}$ with $\Omega_{\text {can }}$ the canonical 2-form on $T^{*} Q$. Also, the vertical lift of a vector $w_{q} \in T_{q} Q$ along the vector $v_{q} \in T_{q} Q$ is the vector vlift $v_{q}\left(w_{q}\right) \in T_{v_{q}}(T Q)$ defined by

$$
\operatorname{vlift}_{v_{q}}\left(w_{q}\right)=\left.\frac{\mathrm{d}}{\mathrm{d} s}\right|_{s=0}\left(v_{q}+s w_{q}\right) .
$$

In coordinates $\left(q^{i}, v^{i}, \delta q^{i}, \delta v^{i}\right)$ on $T T Q$, the lift of the vector with components $w^{i}$ and with base point $q^{i}$ along the vector $v^{i}$, also with base point $q^{i}$, is the vector with base point $\left(q^{i}, v^{i}\right)$ with components given by $\left(0, w^{i}\right)$. Likewise, the vertical lift of a one form $\beta_{q} \in T_{q}^{*} Q$ along a one form $\alpha_{q} \in T_{q}^{*} Q$ is the vector vlift $\alpha_{q}\left(\beta_{q}\right) \in T_{\alpha_{q}}\left(T^{*} Q\right)$ defined by

$$
\operatorname{vlift}_{\alpha_{q}}\left(\beta_{q}\right)=\left.\frac{\mathrm{d}}{\mathrm{d} s}\right|_{s=0}\left(\alpha_{q}+s \beta_{q}\right) .
$$

In coordinates $\left(q^{i}, \alpha_{i}, \delta q^{i}, \delta \alpha_{i}\right)$ on $T T^{*} Q$, the lift of the one form with components $\beta_{i}$ and with base point $q^{i}$ along the one form $\alpha_{i}$, also with base point $q^{i}$, is the vector with base point $\left(q^{i}, \alpha_{i}\right)$ with components given by $\left(0, \beta_{i}\right)$. For a force map $F: T Q \rightarrow T^{*} Q$, the vertical lift vlift $m(F)$ along $m=\mathbb{F} L$ is defined pointwise at $v_{q} \in T_{q} Q$ to be $\operatorname{vlift}_{m}(F)\left(v_{q}\right)=\operatorname{vlift}_{m\left(v_{q}\right)} F\left(v_{q}\right)$. The notation vlift ${ }_{m} u$ is defined in the same way. See [26] for additional properties of Lagrangian vector fields and $[6]$ for more on the forced case.

3. For a given physical system, the force $F$ in the triple $(L, F, W)$ is an external force. For the purpose of controller design, the force $F$ can also be used as a design parameter. As an example of what this means, we consider a gyroscopic force $F_{\mathrm{gr}}$. By definition, gyroscopic forces preserve the energy $E$ of the system. Hence, the following should be satisfied:

$$
\frac{\mathrm{d}}{\mathrm{d} t} E=\left\langle\dot{q}, F_{\mathrm{gr}}\right\rangle=0
$$

Specifically, this holds if $F_{\mathrm{gr}}$ can be written in the following form:

$$
F_{\mathrm{gr}}(q, \dot{q})=S(q, \dot{q}) \dot{q}
$$

where $S(q, \dot{q})$ is a linear map of $T_{q} Q$ to $T^{*} Q$ that satisfies $S(q, \dot{q})^{T}=-S(q, \dot{q})$. In the special case that $S=\left(S_{i j}\right)$ depends only on $q$ and satisfies the following integrability condition,

$$
\frac{\partial S_{i k}(q)}{\partial q^{l}}+\frac{\partial S_{k l}(q)}{\partial q^{i}}+\frac{\partial S_{l i}(q)}{\partial q^{k}}=0
$$


then there are functions $I_{i}(q)$ satisfying

$$
S_{i k}(q)=\frac{\partial I_{i}(q)}{\partial q^{k}}-\frac{\partial I_{k}(q)}{\partial q^{i}}
$$

such that we can incorporate the gyroscopic force $F(q, \dot{q})=S(q, \dot{q}) \dot{q}$ into the Lagrangian as follows: $\left(L, F_{\mathrm{gr}}+\right.$ $F, W) \stackrel{L}{\sim}\left(L+I_{i}(q) \dot{q}^{i}, F, W\right)$. However, one does not have to restrict to such special $S$ 's because the skewsymmetry of $S$ is the only property we need for the gyroscopic force from the energy-conservation point of view. This is essentially the same as using almost Poisson structures by not enforcing the Jacobi-identity condition, which will be discussed in the next section on controlled Hamiltonian systems.

4. Notice that any CL system $(L, F, W)$ is CL-equivalent to $(-L,-F, W)$. The equation $(17)$ becomes $u_{1}=-u_{2}$. If an equilibrium is a minimum point of the energy of $(L, F, W)$, then it will be a maximum point of the energy function of $(-L,-F, W)$. Therefore, it is not crucial whether the energy function has a maximum or a minimum at the equilibrium as far as whether or not we can stabilize the equilibrium.

Control Synthesis via CL system. We now discuss how one may apply the concept of CL-equivalence to designing control laws for mechanical system control problems.

The general idea is as follows. Suppose we are given a CL system $\left(L_{1}, F_{1}, W_{1}\right)$ and a given set of desired performance requirements. In general, it is difficult to directly find a control law to meet these performance requirements. Instead, the strategy is that we first find a CL-equivalent (or CL-included) system $\left(L_{2}, F_{2}, W_{2}\right)$ for which one can more easily find a control $u_{2} \in W_{2}$ such that the performance requirements are met for the closed-loop Lagrangian system $\left(L_{2}, F_{2}, u_{2}\right)$. The control $u_{1}$ can then be calculated according to (17).

For example, the problem of asymptotic stabilization of a desired equilibrium of a given system $\left(L_{1}, F_{1}, W_{1}\right)$ can be stated as follows. Given the CL system $\left(L_{1}, F_{1}, W_{1}\right)$, find a CL-equivalent (or CL system-included) system $\left(L_{2}, F_{2}, W_{2}\right)$ for which one can find a control $u_{2} \in W_{2}$ such that the equilibrium is asymptotically stable for the closed-loop Lagrangian system $\left(L_{2}, F_{2}, u_{2}\right)$. Then, by Proposition 2.4 , the equilibrium is also asymptotically stable in the closed-loop system $\left(L_{1}, F_{1}, u_{1}\right)$ with $u_{1}$ given by $(17)$.

We might also want to ask for a parameterized family of equivalent systems $\left(L_{2}, F_{2}, W_{2}\right)$ satisfying the above so that there is room for tuning, increasing region of attraction, or otherwise improving performance.

The Inverted Pendulum on a Cart. We now apply the theory to the example of an inverted pendulum on a cart and re-derive the result in Section VI of [3] (here, we assume the inclination angle of the slope is zero). The purpose is not to extend in any way these previous results, but rather to use this example in a pedagogical way to illustrate the ideas that have been presented so far in the context of a known example; see Figure 1.

To conform to the notation in the present paper, we shall use $q=\left(q^{1}, q^{2}\right)$ in place of $(\theta, s)$ in [3]. Let $\left(L_{1}, F_{1}=0, W_{1}\right)$ be the inverted pendulum system with the Lagrangian

$$
L_{1}(q, \dot{q})=\frac{1}{2} \alpha\left(\dot{q}^{1}\right)^{2}+\beta \cos \left(q^{1}\right) \dot{q}^{1} \dot{q}^{2}+\frac{1}{2} \gamma\left(\dot{q}^{2}\right)^{2}-D \cos \left(q^{1}\right)
$$

where $\alpha, \beta, \gamma, D$ are positive real numbers (given in terms of the notation of the figure by $\alpha=m l^{2}, \beta=m l, \gamma=$ $M+m$ and $D=m g l)$. Here, $W_{1}$ is spanned by $\mathbf{d} q^{2}$; that is, the matrix $[01]^{T}$. Let $\left(L_{2}, F_{2}, W_{2}\right)$ be a candidate CL system equivalent to $\left(L_{1}, 0, W_{1}\right)$, where the Lagrangian is

$$
L_{2}(q, \dot{q})=\frac{1}{2} m_{2}(\dot{q}, \dot{q})-V_{2}(q)
$$

with

$$
m_{2}(q)=\left[\begin{array}{ll}
m_{11} & m_{12} \\
m_{12} & m_{22}
\end{array}\right]
$$


and the gyroscopic force

$$
F_{2}(q, \dot{q})=\left[\begin{array}{cc}
0 & -f_{1}(q) \dot{q}^{1}-f_{2}(q) \dot{q}^{2} \\
f_{1}(q) \dot{q}^{1}+f_{2}(q) \dot{q}^{2} & 0
\end{array}\right]\left[\begin{array}{c}
\dot{q}^{1} \\
\dot{q}^{2}
\end{array}\right] .
$$

It is reasonable to make the gyroscopic force $F_{2}$ be quadratic in $\dot{q}$ because every term containing $\dot{q}$ in $\mathcal{E} \mathcal{L}\left(L_{2}\right)$ is quadratic in $\dot{q}$. Notice $W_{1}^{\circ}=[10]$. The equation (18) becomes the following:

$$
\begin{aligned}
& 0=A(q) \frac{\partial m_{11}}{\partial q^{1}}+B(q)\left(2 \frac{\partial m_{12}}{\partial q^{1}}-\frac{\partial m_{11}}{\partial q^{2}}-2 f_{1}\right) \\
& 0=A(q)\left(\frac{\partial m_{11}}{\partial q^{2}}+f_{1}\right)+B(q)\left(\frac{\partial m_{22}}{\partial q^{1}}-f_{2}\right) \\
& 0=A(q)\left(2 \frac{\partial m_{12}}{\partial q^{2}}-\frac{\partial m_{22}}{\partial q^{1}}+2 f_{2}\right)+B(q) \frac{\partial m_{22}}{\partial q^{2}}
\end{aligned}
$$

with $A=\left(\alpha m_{22}-\beta \cos \left(q^{1}\right) m_{12}\right)$ and $B=\left(\beta \cos \left(q^{1}\right) m_{11}-\alpha m_{12}\right)$. We can regard $f_{1}$ and $f_{2}$ as free parameters. In order to reproduce the results of [3], we set $f_{1}=f_{2}=0$. However, in such an example as the ball-andbeam system, the usage of this gyroscopic force is crucial (see [29] with the main result of the current paper in mind). One can try to directly solve PDE's in $(22-24)$ as in [1]. However, it is sometimes easier to make some assumptions to reduce the PDE's to a set of ODE's as follows. We assume that $m_{11}$ depends on $q^{1}$ only and $m_{12}$ and $m_{22}$ are of the following form just as in the original system:

$$
m_{12}\left(q^{1}\right)=b \cos \left(q^{1}\right) ; \quad m_{22}=d,
$$

with $b, d \in \mathbb{R}$. Then (23) and (24) are automatically satisfied and (22) becomes

$$
\left(\alpha d-\beta b \cos ^{2}\left(q^{1}\right)\right) m_{11}^{\prime}\left(q^{1}\right)-\left(\beta m_{11}\left(q^{1}\right)-\alpha b\right) 2 b \cos \left(q^{1}\right) \sin \left(q^{1}\right)=0
$$

which can be solved for $m_{11}\left(q^{1}\right)$ as follows:

$$
m_{11}\left(q^{1}\right)=\alpha(a d+b / \beta)-a b \beta \cos ^{2}\left(q^{1}\right)
$$

with $a \in \mathbb{R}$. With this solution, the equation (19) can be written as

$$
\frac{-D(b+a d \beta)}{\beta} \sin \left(q^{1}\right)=\frac{\partial V_{2}}{\partial q^{1}}+a \beta \cos \left(q^{1}\right) \frac{\partial V_{2}}{\partial q^{2}}
$$

which can be solved for $V_{2}$ as follows:

$$
V_{2}\left(q^{1}, q^{2}\right)=D(a d+b / \beta) \cos \left(q^{1}\right)+V_{\epsilon}\left(q^{2}-a \beta \sin \left(q^{1}\right)\right),
$$

with $V_{\epsilon}$ an arbitrary function. The control subbundle $W_{2}$ is computed as follows:

$$
W_{2}=\operatorname{span}\left\{\left(\alpha d-\beta b \cos ^{2}\left(q^{1}\right)\right)\left(\mathbf{d} q^{2}-a \beta \cos \left(q^{1}\right) \mathbf{d} q^{1}\right)\right\} .
$$

For simplicity, let us choose a quadratic function for $V_{\epsilon}$ such that $V_{2}$ becomes

$$
V_{2}\left(q^{1}, q^{2}\right)=D(a d+b / \beta) \cos \left(q^{1}\right)+\frac{1}{2} \epsilon\left(q^{2}-a \beta \sin \left(q^{1}\right)\right)^{2} .
$$


The total energy of the CL system $\left(L_{2}, F_{2}, W_{2}\right)$ is given by

$$
\begin{aligned}
E_{2}=\frac{1}{2}\left(\alpha(a d+b / \beta)-a b \beta \cos ^{2}\left(q^{1}\right)\right)\left(\dot{q}^{1}\right)^{2}+b \cos \left(q^{1}\right) \dot{q}^{1} \dot{q}^{2}+\frac{1}{2} d\left(\dot{q}^{2}\right)^{2} & \\
& +D(a d+b / \beta) \cos \left(q^{1}\right)+\frac{1}{2} \epsilon\left(q^{2}-a \beta \sin \left(q^{1}\right)\right)^{2} .
\end{aligned}
$$

One can check that the energy $E_{2}$ has a minimum at $(0,0,0,0)$ in the set

$$
R:=\left\{\left(q^{1}, q^{2}, \dot{q}^{1}, \dot{q}^{2}\right) \mid \alpha d<\beta b \cos ^{2}\left(q^{1}\right)\right\} \subset\left(-\frac{\pi}{2}, \frac{\pi}{2}\right) \times \mathbb{R}^{3}
$$

if the following holds:

$$
d>0, \quad \epsilon>0, \quad a<0, \quad \alpha d / \beta<b<-a d \beta .
$$

Notice that the condition $\alpha d / \beta<b$ guarantees the constant nonzero rank of $W_{2}$. One can achieve asymptotic stabilization of the equilibrium by choosing a dissipative input $u_{2} \in W_{2}$ as follows:

$$
u_{2}=-c\left(\mathbf{d} q^{2}-a \beta \cos \left(q^{1}\right) \mathbf{d} q^{1}\right) \otimes\left(\mathbf{d} q^{2}-a \beta \cos \left(q^{1}\right) \mathbf{d} q^{1}\right)
$$

with $c>0$. In coordinates,

$$
u_{2}\left(q^{1}, q^{2}, \dot{q}^{1}, \dot{q}^{2}\right)=-c\left(\dot{q}^{2}-a \beta \cos \left(q^{1}\right) \dot{q}^{1}\right)\left[\begin{array}{c}
-a \beta \cos \left(q^{1}\right) \\
1
\end{array}\right] .
$$

The asymptotic stabilization can be straightforwardly proved by the application of LaSalle's theorem (see, for example [23]). By choosing $a, b, \epsilon$ with $|a|,|b| \gg 1,|\epsilon| \ll 1$, one can get a large region of attraction ${ }^{5}$. Notice that we can make the set $R$ in $(25)$ as close to $\left(-\frac{\pi}{2}, \frac{\pi}{2}\right) \times \mathbb{R}^{3}$ as possible, with a large value of $b$.

By Proposition 2.4, the closed-loop system $\left(L_{1}, 0, u_{1}\right)$ with $u_{1}$ obeying $(17)$ has the origin as an asymptotically stable equilibrium in a large region of attraction. Explicitly, $u_{1}$ is given by

$$
u_{1}=\frac{(b \gamma-d \beta)\left(\alpha\left(\dot{q}^{1}\right)^{2}-D \cos \left(q^{1}\right)\right) \sin \left(q^{1}\right)+\left(\alpha \gamma-\beta^{2} \cos ^{2}\left(q^{1}\right)\right)\left(c\left(a \beta \cos \left(q^{1}\right) \dot{q}^{1}-\dot{q}^{2}\right)+\epsilon\left(a \beta \sin \left(q^{1}\right)-q^{2}\right)\right)}{d \alpha-b \beta \cos ^{2}\left(q^{1}\right)} \mathbf{d} q^{2} .
$$

This control law $u_{1}$ is essentially the same as the control law (48) in [3] with $\psi=0$.

Simplified Matching. We review some of the results in [3] in the framework of the current paper. Let $Q=S \times G$ be the configuration space where $G$ is an Abelian Lie group, and let $q=(x, \theta)=\left(x^{\alpha}, \theta^{a}\right) \in S \times G$ be a chosen set of coordinates.

Let $\left(x_{e}, \theta_{e}\right)$ be the equilibrium of interest. Since we will look at the dynamics in a neighborhood of an equilibrium, one may assume that $S$ and $G$ are just Euclidean spaces (a neighborhood does not necessarily mean that it is small in size).

Consider a given CL system of the form $(L, 0, W)$ (so there are no external forces), where $L$ has the form

$$
L=\frac{1}{2} g_{\alpha \beta}(x) \dot{x}^{\alpha} \dot{x}^{\beta}+g_{\alpha a}(x) \dot{x}^{\alpha} \dot{\theta}^{a}+\frac{1}{2} g_{a b} \dot{\theta}^{a} \theta^{b}-V(x)
$$

where we assume that $g_{\alpha \beta}, g_{\alpha a}, V$ depend on $x$ only and $g_{a b}$ is constant. Assume also that the control subbundle

\footnotetext{
${ }^{5}$ In the motivating example in Section 1 , we chose $a=-200, b=100, \epsilon=10^{-5}, c=1$.
} 
is such that the control forces are in the group direction; that is, $W$ is given by $W=0 \oplus T^{*} G \subset T^{*} Q$, i.e.,

$$
W(q)=\text { the subspace of } T_{q}^{*} Q \text { spanned by the columns of }\left[\begin{array}{c}
O \\
I_{G}
\end{array}\right]
$$

where $I_{G}$ is the identity matrix of dimension $\operatorname{dim} G$.

Motivated by the constructive approach for this class of systems given in [3] and references therein, we consider a second CL system $\left(L_{\tau, \sigma, \rho, \epsilon}, 0, W_{\tau, \sigma, \rho, \epsilon}\right)$ given by

$$
\begin{aligned}
L_{\tau, \sigma, \rho, \epsilon}=\frac{1}{2}\left(g_{\alpha \beta}+\rho\left(1-\frac{1}{\sigma}\right)\left(1-\frac{1}{\sigma}-\frac{1}{\rho}\right) g_{\alpha a} g^{a b} g_{b \beta}\right) \dot{x}^{\alpha} \dot{x}^{\beta} & \\
& +\rho\left(1-\frac{1}{\sigma}\right) g_{\alpha a} \dot{x}^{\alpha} \dot{\theta}^{a}+\frac{1}{2} \rho g_{a b} \dot{\theta}^{a} \dot{\theta}^{b}-V\left(x^{\alpha}\right)-V_{\epsilon}\left(x^{\alpha}, \theta^{a}\right),
\end{aligned}
$$

and

$$
\begin{aligned}
& W_{\tau, \sigma, \rho, \epsilon}=\left\langle\mathbf{d} \theta^{a}+\left(1-\frac{1}{\sigma}-\frac{1}{\rho}\right) g^{a c} g_{c \alpha} \mathbf{d} x^{\alpha} \mid a=1, \ldots, \operatorname{dim} G\right\rangle \\
& =\text { the subbundle spanned by the columns of }\left[\left(\begin{array}{c}
\left(1-\frac{1}{\sigma}-\frac{1}{\rho}\right) g_{\alpha d} g^{d a} \\
I_{G}
\end{array}\right]\right.
\end{aligned}
$$

where $\sigma, \rho \in \mathbb{R}$ are free parameters.

To follow the notation in Section 2, let $\left(L_{1}, 0, W_{1}\right)=\left(L_{\tau, \sigma, \rho, \epsilon}, 0, W_{\tau, \sigma, \rho, \epsilon}\right)$ and $\left(L_{2}, 0, W_{2}\right)=(L, 0, W)$. First we examine the condition ELM-1. One computes

$$
m_{2} m_{1}^{-1}\left[\begin{array}{c}
O \\
I_{G}
\end{array}\right]=\left[\begin{array}{c}
\left(1-\frac{1}{\sigma}-\frac{1}{\rho}\right) g_{\alpha d} g^{d a} \\
I_{G}
\end{array}\right]\left[g_{a b}-\left(1-\frac{1}{\sigma}\right) g_{a \alpha} g^{\alpha \beta} g_{\beta b}\right]\left[\rho B^{b e}\right]
$$

where $B^{a b}$ is the inverse matrix of $B_{a b}:=g_{a b}-g_{a \alpha} g^{\alpha \beta} g_{\beta b}$. For ELM-1 to hold, the following should hold: $\rho \neq 0$ and

$$
\left[g_{a b}-\left(1-\frac{1}{\sigma}\right) g_{a \alpha} g^{\alpha \beta} g_{\beta b}\right] \text { is invertible. }
$$

It is an easy linear algebra exercise to show that the matrix $\left[g_{a b}-h g_{a \alpha} g^{\alpha \beta} g_{\beta b}\right]$ is invertible for $h \in \mathbb{R}$ if and only if the matrix $\left[g_{\alpha \beta}-h g_{\alpha a} g^{a b} g_{b \beta}\right]$ is invertible. In [3], we chose $\sigma$ and $\rho$ satisfying

$$
1-\frac{1}{\sigma}>\max \left\{\lambda\left|\operatorname{det}\left[g_{\alpha \beta}-\lambda g_{\alpha a} g^{a b} g_{b \beta}\right]\right|_{x=x_{e}}=0\right\}
$$

and $\rho<0$ in order to achieve stability of the closed-loop system under the assumption that the matrix $\left[g_{a \alpha}\left(x_{e}\right)\right]$ is one to one. With this choice of $\sigma$ and $\rho$, one can see from $(27)$ that $m_{2} m_{1}^{-1}\left(W_{1}\right)=W_{2}$, i.e., ELM-1 holds. Notice that the annihilator $W_{1}^{\circ}$ of $W_{1}$ is spanned by the row vectors of the matrix

$$
K:=\left[I_{G}, \quad-\left(1-\frac{1}{\sigma}-\frac{1}{\rho}\right) g_{\alpha d} g^{d a}\right] .
$$

In this case ELM-2 can be written as

$$
K\left[\mathcal{E} \mathcal{L}\left(L_{1}\right)-m_{1} m_{2}^{-1} \mathcal{E} \mathcal{L}\left(L_{2}\right)\right]=O,
$$


which holds if and only if

$$
-\left(\frac{\partial V}{\partial \theta^{a}}+\frac{\partial V_{\epsilon}}{\partial \theta^{a}}\right)\left(1-\frac{1}{\sigma}-\frac{1}{\rho}\right) g^{a d} g_{d \alpha}+\frac{\partial V_{\epsilon}}{\partial x^{\alpha}}=0
$$

which is equation (16) in [3].

Letting $u_{1}=u^{\text {diss }}$ times the basis vector given in (26), equation (17) can then be solved for $u_{2}$, which is the control for the originally given system $(L, 0, W)$. The expression for $u_{2}$ derived from (17) is the same as equation (22) in [3]. In the same paper, the energy function of the CL system $\left(L_{\tau, \sigma, \rho, \epsilon}, 0, W_{\tau, \sigma, \rho, \epsilon}\right)$ has a maximum at the equilibrium point and in that paper there is a detailed computation showing the asymptotic stability of the equilibrium in the closed-loop system. Also, in [3] use is made of the integrability of $W_{2}$ to choose another set of local coordinates convenient for an asymptotic stability analysis.

\section{Controlled Hamiltonian systems}

In this section we formally define a controlled Hamiltonian $(\mathrm{CH})$ system analogously to a controlled Lagrangian system. First we discuss almost Poisson structures.

Almost Poisson Structure. Following [12], we define an almost Poisson tensor $B$ on $T^{*} Q$ to be a skewsymmetric $(2,0)$-tensor on $T^{*} Q$. Its almost Poisson bracket $\{\}:, \mathcal{F}\left(T^{*} Q\right) \times \mathcal{F}\left(T^{*} Q\right) \rightarrow \mathcal{F}\left(T^{*} Q\right)$ (where $\mathcal{F}(M)$ is the space of smooth real-valued functions on the manifold $M)$ is defined as

$$
\{F, G\}=B(\mathbf{d} F, \mathbf{d} G)
$$

for $F, G \in \mathcal{F}\left(T^{*} Q\right)$. Then $\{$,$\} satisfies the following properties:$

(i) $\{F, G\}=-\{G, F\}$,

(ii) $\{F+G, H\}=\{F, H\}+\{G, H\}$,

(iii) $\{F G, H\}=F\{G, H\}+G\{F, H\}$

for $F, G, H \in \mathcal{F}\left(T^{*} Q\right)$. It is not necessary that it satisfy the Jacobi identity:

$$
\{F,\{G, H\}\}+\{G,\{H, F\}\}+\{H,\{F, G\}\}=0
$$

for $F, G, H \in \mathcal{F}\left(T^{*} Q\right)$. In coordinates, the almost Poisson tensor $B$ can be written in terms of its action on the coordinate functions:

$$
B(q, p)=\left[\begin{array}{ll}
\left\{q^{i}, q^{j}\right\} & \left\{q^{i}, p_{j}\right\} \\
\left\{p_{i}, q^{j}\right\} & \left\{p_{i}, p_{j}\right\}
\end{array}\right] .
$$

The induced map $B^{\sharp}: T^{*} T^{*} Q \rightarrow T T^{*} Q$ is defined as

$$
B\left(\alpha_{z}, \beta_{z}\right)=\left\langle\alpha_{z}, B^{\sharp}(z) \beta_{z}\right\rangle
$$

for $\alpha_{z}, \beta_{z} \in T_{z}^{*} T^{*} Q$.

It is well-known that almost Poisson structures arise in nonholonomic mechanics; see, e.g. [24,36] and references therein. The geometric way of expressing the Jacobi identity of the $\{$,$\} in terms of B$ is to use the Schouten bracket (see Chap. 10 of [26] for more details).

Controlled Hamiltonian Systems. The Hamiltonian analog of a CL system is defined as follows:

Definition 3.1. A controlled Hamiltonian system (CH system) is a quadruple $(H, B, F, W)$ where $H$ : $T^{*} Q \rightarrow \mathbb{R}$ is the Hamiltonian function, $B$ is an almost Poisson tensor, $F: T^{*} Q \rightarrow T^{*} Q$ is the (external force) fiber-preserving map, and $W \subset T^{*} Q$ is the (control) subbundle. 
Sometimes, $W$ denotes the set of bundle maps from $T^{*} Q$ to $W$. As on the Lagrangian side, when we choose a specific control $u: T^{*} Q \rightarrow W$, then we call the quadruple $(H, B, F, u)$ a closed-loop Hamiltonian system. The vector field $X_{(H, B, F, u)}$ of the closed-loop system $(H, B, F, u)$ is given by

$$
X_{(H, B, F, u)}=B^{\sharp} \mathbf{d} H+\operatorname{vift}(F)+\operatorname{vlift}(u)
$$

with a control $u: T^{*} Q \rightarrow W$, where the vertical lift operator vlift $: T^{*} Q \times T^{*} Q \rightarrow T T^{*} Q$ is defined as follows: for $\alpha_{q}, \beta_{q} \in T_{q}^{*} Q$

$$
T_{\alpha_{q}} T^{*} Q \ni \operatorname{vlift}\left(\beta_{q}, \alpha_{q}\right)=\left.\frac{\mathrm{d}}{\mathrm{d} s}\right|_{s=0}\left(\alpha_{q}+s \beta_{q}\right)
$$

and $\operatorname{vlift}(F): T^{*} Q \rightarrow T T^{*} Q$ is defined by

$$
\operatorname{vlift}(F)\left(\alpha_{q}\right)=\operatorname{vlift}\left(F\left(\alpha_{q}\right), \alpha_{q}\right)
$$

for $\alpha_{q} \in T_{q}^{*} Q$ and $\operatorname{vift}(u)$ is defined in a similar manner. For a subbundle $W$ of $T^{*} Q$, define

$$
\operatorname{vlift}(W)=\bigcup_{q \in Q}\left\{\operatorname{vlift}\left(\beta_{q}, \alpha_{q}\right) \mid \alpha_{q} \in T_{q} Q, \beta_{q} \in W_{q}\right\} .
$$

See also Remark 2 in Section 2.

Hamiltonian Matching. Suppose we have two controlled Hamiltonian systems $\left(H_{i}, B_{i}, F_{i}, W_{i}\right), i=1,2$.

Definition 3.2. We say that these systems satisfy the Hamiltonian matching conditions if

HM-1: $W_{1}=W_{2}$,

HM-2: $\operatorname{Im}\left[\left(B_{1}^{\sharp} \mathbf{d} H_{1}+\operatorname{vlift}\left(F_{1}\right)\right)-\left(B_{2}^{\sharp} \mathbf{d} H_{2}+\operatorname{vift}\left(F_{2}\right)\right)\right] \subset \operatorname{vlift}\left(W_{1}\right)$.

In addition we say that two Hamiltonian systems are $\mathbf{C H}$-equivalent if HM-1 and HM-2 hold for the systems. We use the symbol, $\stackrel{H}{\sim}$ for this equivalence relation.

Proposition 3.3. Suppose that the two $C H$ systems $\left(H_{1}, B_{1}, F_{1}, W_{1}\right)$ and $\left(H_{2}, B_{2}, F_{2}, W_{2}\right)$ are CH-equivalent. Then the two closed-loop systems $\left(H_{1}, B_{1}, F_{1}, u_{1}\right)$ and $\left(H_{2}, B_{2}, F_{2}, u_{2}\right)$ produce the same vector fields if and only if the controls $u_{1}$ and $u_{2}$ satisfy

$$
\operatorname{vlift}\left(u_{2}\right)=\operatorname{vlift}\left(u_{1}\right)+\left(B_{1}^{\sharp} \mathbf{d} H_{1}+\operatorname{vlift}\left(F_{1}\right)\right)-\left(B_{2}^{\sharp} \mathbf{d} H_{2}+\operatorname{vift}\left(F_{2}\right)\right) .
$$

Proof. Just consider the following equation:

$$
X_{\left(H_{1}, B_{1}, F_{1}, u_{1}\right)}=X_{\left(H_{2}, B_{2}, F_{2}, u_{2}\right)} .
$$

One can also define a partial order, $\mathrm{CH}$-inclusion in the class of $\mathrm{CH}$ systems on $T^{*} Q$ as follows.

Definition 3.4. A CH system $\left(H_{1}, B_{1}, F_{1}, W_{1}\right)$ is said to include another $\mathrm{CH}$ system $\left(H_{2}, B_{2}, F_{2}, W_{2}\right)$ if the following holds:

HI-1: $W_{1} \supset W_{2}$,

HI-2: $\operatorname{Im}\left[\left(B_{1}^{\sharp} \mathbf{d} H_{1}+\operatorname{vlift}\left(F_{1}\right)\right)-\left(B_{2}^{\sharp} \mathbf{d} H_{2}+\operatorname{vlift}\left(F_{2}\right)\right)\right] \subset \operatorname{vlift}\left(W_{1}\right)$.

If $\left(H_{1}, B_{1}, F_{1}, W_{1}\right)$ includes $\left(H_{2}, B_{2}, F_{2}, W_{2}\right)$, then for any choice of control $u_{2}: T^{*} Q \rightarrow W_{2}$, there exists a control $u_{1}: T^{*} Q \rightarrow W_{1}$ satisfying (28) such that the two closed-loop systems with these controls produce the same equations of motion. 
Simple CH Systems. The definition of a simple Hamiltonian control system is slightly more subtle than its Lagrangian counterpart.

Definition 3.5. A CH system $(H, B, F, W)$ is called simple when the Hamiltonian function has the form kinetic plus potential energy:

$$
H(q, p)=\frac{1}{2}\left\langle p, m^{-1}(q) p\right\rangle+V(q)
$$

where $m$ is a nondegenerate symmetric $(0,2)$-tensor and the almost Poisson tensor $B$ is nondegenerate and has the form:

$$
B(q, p)=\left[\begin{array}{cc}
O & K(q)^{T} \\
-K(q) & J(q, p)
\end{array}\right]
$$

in cotangent coordinates $(q, p)$ on $T^{*} Q$ where $K, J$ are $n \times n$ matrices with $n=\operatorname{dim} Q$.

One can check that the statement that $B$ has the form (30) is independent of the choice of cotangent bundle coordinates for $T^{*} Q$. We call almost Poisson tensors of form (30) with $K$ invertible simple.

Decomposition of Simple Almost Poisson Tensors. Now, we define a decomposition of simple almost Poisson tensors in the following way. Let $B$ be a given simple almost Poisson tensor. The following relation

$$
\operatorname{vift}\left(\psi_{B}\right)=\left(B^{\sharp}\right)^{*} \circ \Theta
$$

defines a unique $\psi_{B} \in \Gamma\left(\operatorname{Aut}\left(T^{*} Q\right)\right)$. Here, $\Gamma\left(\operatorname{Aut}\left(T^{*} Q\right)\right)$ denotes sections of the automorphism bundle; that is, and element of $\Gamma\left(\operatorname{Aut}\left(T^{*} Q\right)\right)$ assigns to each $q \in Q$, an invertible linear transformation of $T_{q}^{*} Q$ to itself. Also, $\Theta$ denotes the canonical one form on $T^{*} Q$, and $\left(B^{\sharp}\right)^{*}: T^{*} T^{*} Q \rightarrow T T^{*} Q$ is the dual of $B^{\sharp}: T^{*} T^{*} Q \rightarrow T T^{*} Q$. Suppose $B$ is given by (30) in coordinates. Then,

$$
B^{\sharp}(q, p)=K_{j i}(q) \frac{\partial}{\partial p_{j}} \otimes \frac{\partial}{\partial q^{i}}-K_{i j}(q) \frac{\partial}{\partial q^{j}} \otimes \frac{\partial}{\partial p_{i}}+J_{i j}(q, p) \frac{\partial}{\partial p_{j}} \otimes \frac{\partial}{\partial p_{i}} .
$$

Since $\left(B^{\sharp}\right)^{*}(q, p)=-B^{\sharp}(q, p)$, we have

$$
\left(B^{\sharp}\right)^{*} \circ \Theta(q, p)=-B^{\sharp}(q, p)\left(p_{k} \mathrm{~d} q^{k}\right)=K_{i j}(q) p_{j} \frac{\partial}{\partial p_{i}}
$$

which is the vertical lift of $K_{i j}(q) p_{j} \mathrm{~d} q^{i}$ at $(q, p)$. Hence, (31) defines a unique $\psi_{B} \in \Gamma\left(\operatorname{Aut}\left(T^{*} Q\right)\right)$ and its local expression is given by

$$
\psi_{B}(q, p)=(q, K(q) p)
$$

with $B$ given by (30) in coordinates.

Given a simple almost Poisson tensor $B$, we can uniquely decompose $B$ into the two almost Poisson tensors $B_{\mathrm{r}}$ and $B_{\mathrm{gr}}$ as follows:

$$
B=B_{\mathrm{r}}+B_{\mathrm{gr}}
$$

where

$$
B_{\mathrm{r}}=\left(\psi_{B}^{-1}\right)^{*} B_{\mathrm{can}} ; \quad B_{\mathrm{gr}}=B-\left(\psi_{B}^{-1}\right)^{*} B_{\mathrm{can}}
$$


and $B_{\text {can }}$ is the canonical Poisson tensor on $T^{*} Q$. When $B$ is given by (30) in coordinates, we have the following coordinate expressions:

$$
\begin{aligned}
B_{\mathrm{r}}(q, p) & =\left[\begin{array}{cc}
O & K(q)^{T} \\
-K(q) & C_{K}(q, p)
\end{array}\right] \\
B_{\mathrm{gr}}(q, p) & =\left[\begin{array}{lc}
O & O \\
O & J(q, p)-C_{K}(q, p)
\end{array}\right]
\end{aligned}
$$

where

$$
\begin{aligned}
\left(C_{K}\right)_{i j}(q, p) & =-\left\langle K(q)^{-1} p,\left[\left(K(q)^{T}\right)_{i},\left(K(q)^{T}\right)_{j}\right]\right\rangle \\
& =\left(\frac{\partial K_{i s}(q)}{\partial q^{r}} K_{j r}(q)-K_{i r}(q) \frac{\partial K_{j s}(q)}{\partial q^{r}}\right) K^{s l}(q) p_{l}
\end{aligned}
$$

where $\left(K(q)^{T}\right)_{i}$ is the $i$-th column of the matrix $K(q)^{T}$ and [, ] is the Lie bracket. The formula (36) is essentially the same as the equation (19) in [36]. By $(30,32)$ and $(34)$, we have

$$
\psi_{B}=\psi_{B_{\mathrm{r}}} .
$$

Notice that the Poisson tensor $B_{\mathrm{r}}$ satisfies the Jacobi-identity because it is a pull-back of the canonical Poisson bracket.

Construction of Gyroscopic Forces. Given a simple Hamiltonian system $\left(H, B=B_{\mathrm{r}}+B_{\mathrm{gr}}, F, W\right)$, the simple (degenerate) almost Poisson tensor $B_{\mathrm{gr}}$ and the Hamiltonian $H$ defines a gyroscopic force $F_{\mathrm{gr}}: T^{*} Q \rightarrow T^{*} Q$ by the following relation:

$$
\operatorname{vlift}\left(F_{\mathrm{gr}}\right)=\left(B_{\mathrm{gr}}\right)^{\sharp} \mathrm{d} H \text {. }
$$

By (35), in coordinates

$$
\left(B_{\mathrm{gr}}\right)^{\sharp} \mathrm{d} H(q, p)=\left(\left(J-C_{K}\right)_{i j}(q, p) \frac{\partial H(q, p)}{\partial p_{j}}\right) \frac{\partial}{\partial p_{i}}
$$

which is the vertical lift of $\left(\left(J-C_{K}\right)_{i j}(q, p) \frac{\partial H(q, p)}{\partial p_{j}}\right) \mathrm{d} q^{i}$ at $(q, p)$. Hence, (38) defines the unique force $F_{\mathrm{gr}}$ : $T^{*} Q \rightarrow T^{*} Q$, which is locally written as

$$
F_{\mathrm{gr}}(q, p)=\left(q,\left(J-C_{K}\right)_{i j}(q, p) \frac{\partial H(q, p)}{\partial p_{j}}\right) .
$$

The reason we call $F_{\text {gr }}$ the gyroscopic force is that it does not change the Hamiltonian $H$ in the following sense

$$
\operatorname{vlift}\left(F_{\mathrm{gr}}\right)[H]=\mathrm{d} H\left(\left(B_{\mathrm{gr}}\right)^{\sharp} \mathrm{d} H\right)=B_{\mathrm{gr}}(\mathrm{d} H, \mathrm{~d} H)=0
$$

by the skew-symmetry of $B_{\mathrm{gr}}$. The dynamics with gyroscopic forces still gives conservation of energy.

CH-Equivalence for Simple Systems. This decomposition of simple almost Poisson tensors simplifies the class of simple Hamiltonian systems under the $\mathrm{CH}$-equivalence relation. Suppose that we are given a simple Hamiltonian system $\left(H, B=B_{\mathrm{r}}+B_{\mathrm{gr}}, F, W\right)$. Then (38) implies

$$
B^{\sharp} \mathbf{d} H+\operatorname{vlift}(F)=\left(B_{\mathrm{r}}\right)^{\sharp} \mathbf{d} H+\operatorname{vlift}\left(F_{\mathrm{gr}}+F\right) .
$$


Therefore the simple Hamiltonian system $\left(H, B=B_{\mathrm{r}}+B_{\mathrm{gr}}, F, W\right)$ is CH-equivalent to the simple Hamiltonian system $\left(H, B_{\mathrm{r}}, F_{\mathrm{gr}}+F, W\right)$ where $F_{\mathrm{gr}}$ is given by (38). By (33) and (37),

$$
\psi_{B_{\mathrm{r}}}^{*} B_{\mathrm{r}}=\psi_{B}^{*} B_{\mathrm{r}}=B_{\mathrm{can}} .
$$

This proves the following result:

Proposition 3.6. A given simple $C H$ system $\left(H, B=B_{\mathrm{r}}+B_{\mathrm{gr}}, F, W\right)$ is $C H$-equivalent to the $C H$ system $\left(H, B_{\mathrm{r}}, F_{\mathrm{gr}}+F, W\right)$ where $B=B_{\mathrm{r}}+B_{\mathrm{gr}}$ is the decomposition of $B$ into the regular part and the gyroscopic part and $F_{\mathrm{gr}}: T^{*} Q \rightarrow T^{*} Q$ is determined by the relation $\operatorname{vlift}\left(F_{\mathrm{gr}}\right)=B_{\mathrm{gr}}^{\sharp} \mathrm{d} H$. In particular, $B_{r}$ satisfies (39).

A consequence is

Corollary 3.7. An arbitrary simple $C H$ system is $C H$-equivalent to a simple Hamiltonian system $(H, B, F, W)$ with the Poisson tensor $B$ satisfying the Jacobi identity and $\psi_{B}^{*} B=B_{\text {can }}$. Equivalently, one can say $B=\phi^{*} B_{\text {can }}$ for some $\phi \in \Gamma\left(\operatorname{Aut}\left(T^{*} Q\right)\right)$. In coordinates, $B$ is always of the form

$$
B(q, p)=\left[\begin{array}{cc}
O & K(q)^{T} \\
-K(q) & C_{K}(q, p)
\end{array}\right]
$$

with $C_{K}$ in (36) when $\phi\left(\right.$ or, $\left.\psi_{B}^{-1}\right)$ is in coordinates given by $\phi(q, p)=\left(q, K(q)^{-1} p\right)$.

Proof. A direct consequence of Proposition 3.6 and (34).

We now consider the opposite direction of Proposition 3.6. Let us consider a simple CH system $\left(H, B, F_{\mathrm{gr}}, W\right)$ with $H=\frac{1}{2}\left\langle p, m^{-1} p\right\rangle+V(q)$ and $F_{\mathrm{gr}}$ a gyroscopic force. By definition of the gyroscopic force, $F_{\mathrm{gr}}=\left(F_{\mathrm{gr}}\right)_{i} \mathbf{d} q^{i}$ satisfies

$$
0=\operatorname{vlift}\left(F_{\mathrm{gr}}\right)[H]=\left(F_{\mathrm{gr}}\right){ }_{i} m^{i j} p_{j}
$$

where $m^{-1}=\left(m^{i j}\right)$. Hence, $\left(F_{\mathrm{gr}}\right)_{i}$ should be of the following form

$$
\left(F_{\mathrm{gr}}\right)_{i}(q, p)=p_{l} S^{l k}(q, p) m_{k i}(q), \quad S^{l k}=-S^{k l} .
$$

Hence, we have proved the following:

Proposition 3.8. Given a simple $C H$ system $\left(H, B, F+F_{\mathrm{gr}}, W\right)$ with $F_{\mathrm{gr}}$ a gyroscopic force, the following holds:

1. Fr has the form:

$$
F_{\mathrm{gr}}(q, p)=p_{l} S^{l k}(q, p) m_{k i}(q) \mathbf{d} q^{i}, \quad S^{l k}=-S^{k l}
$$

for some functions $S^{i j}(q, p)$, where $m_{i j}$ is the mass matrix of $H$.

2. We have

$$
\left(H, B, F+F_{\mathrm{gr}}, W\right) \stackrel{H}{\sim}(H, B+\tilde{B}, F, W)
$$

where

$$
\tilde{B}(q, p)=m_{i k}(q) S^{k l}(q, p) m_{l j}(q) \frac{\partial}{\partial p_{i}} \otimes \frac{\partial}{\partial p_{j}} .
$$

Proof. One needs to check that $B+\tilde{B}$ is a simple Poisson tensor, which is readily done.

Proposition 3.6 and Proposition 3.8 show that the failure of the Jacobi identity of the Poisson bracket of a Poisson bracket of a $\mathrm{CH}$ system is directly related to gyroscopic forces. 
Pull-back Systems. The concept of pull-back systems will be useful in the next section in showing the equivalence of the CL system method and the $\mathrm{CH}$ system method. This notion is a technical device that is needed for the proofs later and can be omitted on first reading. Consider a $\mathrm{CH}$ system $(H, B, F, W)$ and $\phi \in \Gamma\left(\operatorname{Aut}\left(T^{*} Q\right)\right)$. Then, the pull-back system $\phi^{*}(H, B, F, W)$ is defined to be the associated CH system $\left(\phi^{*} H, \phi^{*} B, \phi^{*} F, \phi^{*} W\right)$, where

$$
\phi^{*} H=H \circ \phi \quad \text { and } \quad\left(\phi^{*} B\right)(z)\left(\alpha_{z}, \beta_{z}\right)=B(w)\left(T_{w}^{*}\left(\phi^{-1}\right) \alpha_{z}, T_{w}^{*}\left(\phi^{-1}\right) \beta_{z}\right)
$$

for $\phi(w)=z$ and $\alpha_{z}, \beta_{z} \in T_{z}^{*}\left(T^{*} Q\right)$, and $\phi^{*} F=\phi^{-1} \circ F \circ \phi$. Here, the pull-back notation in $\phi^{*} F$ should be regarded as an action of $\Gamma\left(\operatorname{Aut}\left(T^{*} Q\right)\right)$ on the set of fiber-preserving maps on $T^{*} Q$. Notice also that in the current paper, $\phi^{*} W=\phi^{-1}(W)$ by definition. The notation $\phi^{*} W$ should not be confused with the standard notation of pull-back bundles. When we regard $W$ as the set $\left\{u: T^{*} Q \rightarrow W\right\}$, then $\phi^{*} W$ reads $\phi^{*} W=\left\{\phi^{*} u=\right.$ $\left.\phi^{-1} \circ u \circ \phi \mid u \in W\right\}$. Hence, we will write $\phi^{*} W$ as $\phi^{-1} W \circ \phi$ to respect both interpretations. We claim

$$
\phi^{*} X_{(H, B, F, u)}=X_{\phi^{*}(H, B, F, u)}
$$

It is well-known (or a straightforward computation) that $\left(\phi^{*} B\right)^{\sharp} \mathbf{d}\left(\phi^{*} H\right)=\phi^{*}\left(B^{\sharp} \mathbf{d} H\right)$. We have only to show $\phi^{*}(\operatorname{vlift}(F))=\operatorname{vlift}\left(\phi^{*} F\right)$ where one should be careful that pull-back notation in the left hand side is the usual pull-back of a vector field by a diffeomorphism $\phi$, and the pull-back notation on the right hand side should be understood as $\phi^{-1} \circ F \circ \phi$ as we mentioned before. Indeed, for $w \in T^{*} Q$, we have

$$
\begin{aligned}
\left(\phi^{*}(\operatorname{vift}(F))\right)(w) & =T_{\phi(w)} \phi^{-1} \cdot \operatorname{vlift}(F)(\phi(w)) \\
& =\left.T_{\phi(w)} \phi^{-1} \frac{\mathrm{d}}{\mathrm{d} s}\right|_{s=0}(\phi(w)+s F \circ \phi(w)) \\
& =\left.\frac{\mathrm{d}}{\mathrm{d} s}\right|_{s=0} \phi^{-1}(\phi(w)+s F \circ \phi(w)) \\
& =\left.\frac{\mathrm{d}}{\mathrm{d} s}\right|_{s=0}\left(w+s\left(\phi^{*} F\right)(w)\right) \\
& =\operatorname{vlift}\left(\phi^{*} F\right)(w) .
\end{aligned}
$$

The same relation holds for $u$. One can readily show the following:

Proposition 3.9. Let $\phi \in \Gamma\left(\operatorname{Aut}\left(T^{*} Q\right)\right)$. Then the following hold:

1. The pull-back system via $\phi$ of a simple $C H$ system is also simple.

2. Two $C H$ systems $\left(H_{1}, B_{1}, F_{1}, W_{1}\right)$ and $\left(H_{2}, B_{2}, F_{2}, W_{2}\right)$ are $C H$-equivalent if and only if the corresponding pull-back systems $\phi^{*}\left(H_{1}, B_{1}, F_{1}, W_{1}\right)$ and $\phi^{*}\left(H_{2}, B_{2}, F_{2}, W_{2}\right)$ are CH-equivalent.

In particular, it is useful to have a coordinate expression for $\phi^{*} B$ when $B$ satisfies $\psi_{B}^{*} B=B_{\text {can }}$ for $\psi \in$ $\Gamma\left(\operatorname{Aut}\left(T^{*} Q\right)\right)$. The almost Poisson tensor $B$ is written in coordinates as in (30). Consider $\phi \in \Gamma\left(\operatorname{Aut}\left(T^{*} Q\right)\right)$ with the local coordinate expression $\phi(q, p)=\left(q, D(q)^{-1} p\right)$. Then the pull-back tensor $\phi^{*} B$ is expressed in coordinates as

$$
\left(\phi^{*} B\right)(q, p)=\left[\begin{array}{cc}
O & (D(q) K(q))^{T} \\
-D(q) K(q) & C_{D K}
\end{array}\right]
$$

since

$$
\phi^{*} B=\left(\left(\psi_{B}\right)^{-1} \circ \phi\right)^{*}\left(\psi_{B}\right)^{*} B=\left(\left(\psi_{B}\right)^{-1} \circ \phi\right)^{*} B_{\mathrm{can}} .
$$

Here $\psi_{B}^{-1} \circ \phi(q, p)=\left(q,(D(q) K(q))^{-1} p\right)$ and we use the formula in Corollary 3.7. The equation (40) implies that $\psi_{\phi^{*} B}=\phi^{-1} \circ \psi_{B}$ and $\left(\psi_{\phi^{*} B}\right)^{*} B=B_{\text {can }}$. This proves that a simple CH system $(H, B, F, W)$ with $\psi_{B}^{*} B=B_{\text {can }}$ is pulled back by $\phi \in \Gamma\left(\operatorname{Aut}\left(T^{*} Q\right)\right)$ to the simple $\mathrm{CH}$ system $\phi^{*}(H, B, F, W)$ satisfying $\left(\psi_{\phi^{*} B}\right)^{*}\left(\phi^{*} B\right)=B$ can and $\psi_{\phi^{*} B}=\phi^{-1} \circ \psi_{B}$. 


\section{Equivalence of CL systems and CH systems: Simple mechanical systems}

The goal of this section is to show that for simple mechanical systems, the method of controlled Lagrangians and that of controlled Hamiltonians are equivalent. A more detailed statement is contained in Theorem 4.1 and Corollary 4.2. For this purpose, we will first review the Legendre transformations and then get to the problem of the equivalence of the two methods of CL systems and CH systems.

Frequently, a Hamiltonian system on $T^{*} Q$ induces a Hamiltonian vector field through a canonical symplectic structure (or, canonical Poisson structure) on $T^{*} Q$ before any reduction processes. This is because a Hamiltonian system on $T^{*} Q$ often comes from a Lagrangian system on $T Q$ via a Legendre transformation associated to a given Lagrangian function. Hence if there is more than one Lagrangian function, there can be multiple transformations between $T Q$ and $T^{*} Q$. We will carefully deal with this in this section, too.

Legendre Transformations. Recall that in this paper all the Lagrangians $L$ on $T Q$ and all the Hamiltonians $H$ on $T^{*} Q$ are regular, i.e., $\operatorname{det}\left(\frac{\partial^{2} L}{\partial \dot{q}^{2} \partial \dot{q}^{j}}\right) \neq 0$ and $\operatorname{det}\left(\frac{\partial^{2} H}{\partial p_{i} \partial p_{j}}\right) \neq 0$ for $(q, \dot{q}) \in T Q$ and $(q, p) \in T^{*} Q$. This assumption implies that the Legendre transformation $\mathbb{F} L: T Q \rightarrow T^{*} Q$ (defined in (21)) and the inverse Legendre transformation $\mathbb{F} H: T^{*} Q \rightarrow T Q$ (defined below) are both locally invertible. In fact, we shall assume that the systems are hyperregular; that is, these transformations are globally invertible. This is automatic for simple systems.

It is well-known (see, for example [26]) that a given Lagrangian system $\left(L, F^{L}, W^{L}\right)$ is transformed by the Legendre transformation $\mathbb{F} L$ to the Hamiltonian system $\left(H, B_{\mathrm{can}}, F^{H}, W^{H}\right)$ where

$$
\begin{aligned}
H(\alpha) & =\left\langle\alpha, \mathbb{F} L^{-1}(\alpha)\right\rangle-L \circ \mathbb{F} L^{-1}(\alpha) \quad \text { for } \alpha \in T^{*} Q \\
F^{H} & =F^{L} \circ \mathbb{F} L^{-1} \\
W^{H} & =W^{L} \circ \mathbb{F} L^{-1}
\end{aligned}
$$

where $W^{L} \circ \mathbb{F} L^{-1}$ is understood as $W^{L}$ as a subbundle of $T^{*} Q$ and also understood as the set $\left\{u \circ \mathbb{F} L^{-1} \mid u\right.$ : $\left.T Q \rightarrow W^{L}\right\}$ when we regard $W^{L} \circ \mathbb{F} L^{-1}$ as a set of bundle maps from $T^{*} Q$ to $W$. Namely, the Euler-Lagrange equation

$$
\mathcal{E} \mathcal{L}(L)=F^{L}+u^{L}
$$

with $u^{L}: T Q \rightarrow W^{L}$ is equivalent to $\mathrm{CH}$ vector field

$$
X_{\left(H, B_{\text {can }}, F^{H}, u^{H}\right)}=B_{\text {can }}^{\sharp} \mathbf{d} H+\operatorname{vlift}\left(F^{L} \circ \mathbb{F} L^{-1}\right)+\operatorname{vlift}\left(u^{L} \circ \mathbb{F} L^{-1}\right) .
$$

We now suppose that we are given a Hamiltonian function $H$ on $T^{*} Q$. Then it induces the inverse Legendre transformation $\mathbb{F} H: T^{*} Q \rightarrow T Q$ as follows:

$$
\left\langle\mathbb{F} H\left(\alpha_{q}\right), \beta_{q}\right\rangle=\left.\frac{\mathrm{d}}{\mathrm{d} s}\right|_{s=0} H\left(\alpha_{q}+s \beta_{q}\right)
$$

for $\alpha_{q}, \beta_{q} \in T_{q}^{*} Q$, where the word inverse will be justified later. It is well-known (or easy to check ) that a given $\mathrm{CH}$ system $\left(H, B_{\text {can }}, F^{H}, W^{H}\right)$ is transformed by the inverse Legendre transformation $\mathbb{F} H$ to the CL system $\left(L, F^{L}=F^{H} \circ \mathbb{F} H^{-1}, W^{L}=W^{H} \circ \mathbb{F} H^{-1}\right)$ where $L(v)=\left\langle v, \mathbb{F} H^{-1}(v)\right\rangle-H \circ \mathbb{F} H^{-1}(v)$ for $v \in T Q$. The CH vector field $X_{\left(H, B_{\text {can }}, F^{H}, u^{H}\right)}=B_{\text {can }}^{\sharp} \mathbf{d} H+\operatorname{vlift}\left(F^{H}\right)+\operatorname{vlift}\left(u^{H}\right)$ is equivalent to the Euler-Lagrange equation $\mathcal{E} \mathcal{L}(L)=F^{L}+u^{H} \circ \mathbb{F} H^{-1}$.

Suppose that a given CL system $\left(L, F^{L}, W^{L}\right)$ is transformed by the Legendre transformation $\mathbb{F} L$ to the CH system $\left(H, B_{\text {can }}, F^{H}, W^{H}\right)$. Then $\left(H, B_{\text {can }}, F^{H}, W^{H}\right)$ is transformed back to $\left(L, F^{L}, W^{L}\right)$ by the inverse Legendre transformation $\mathbb{F} H$ since $\mathbb{F} H=\mathbb{F} L^{-1}$ in this case by Proposition 7.4.2 of [26]. One can also start this argument from the Hamiltonian system. 


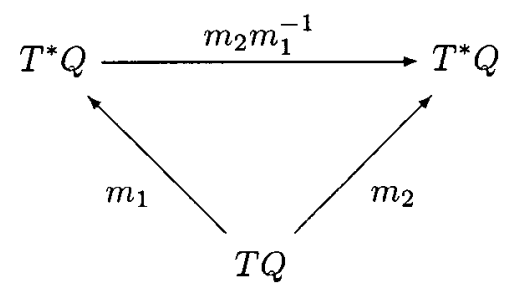

FIGURE 2. Diagram of Legendre transformations.

CH-Equivalence Proves CL-Equivalence. We first show that the matching conditions of simple CL systems can be derived from those of simple $\mathrm{CH}$ systems; the computation involved in this direction is simpler than that involved in showing the other way around. However, the computation carried out here will be also used in the paragraph entitled CL-Equivalence Proves CH-Equivalence. A special case of CH-Equivalence Proves CL-Equivalence was dealt with in [29] and [2] but without the external force $F$. We also improve the foundational setting for the controlled Hamiltonian method. This improved setting will pay off when we consider systems with symmetry and reduction.

Suppose we have two simple CL systems $\left(L_{1}, F_{1}^{L}, W_{1}^{L}\right)$ and $\left(L_{2}, F_{2}^{L}, W_{2}^{L}\right)$ with $L_{1}(q, \dot{q})=\frac{1}{2} m_{1}(\dot{q}, \dot{q})-V_{1}(q)$ and $L_{2}(q, \dot{q})=\frac{1}{2} m_{2}(\dot{q}, \dot{q})-V_{2}(q)$. They define two Legendre transformations $\mathbb{F} L_{1}, \mathbb{F} L_{2}: T Q \rightarrow T^{*} Q$ as follows

$$
\begin{aligned}
& (q, p)=\mathbb{F} L_{1}(q, \dot{q})=\left(q, m_{1}(q) \dot{q}\right), \\
& (q, \tilde{p})=\mathbb{F} L_{2}(q, \dot{q})=\left(q, m_{2}(q) \dot{q}\right) .
\end{aligned}
$$

The CL system $\left(L_{1}, F_{1}^{L}, W_{1}^{L}\right)$ is transformed via $\mathbb{F} L_{1}$ to the $\mathrm{CH}$ system $\left(H_{1}, B_{1}=B_{\text {can }}, F_{1}^{H}=F_{1}^{L} \circ \mathbb{F} L^{-1}, W_{1}^{H}=\right.$ $\left.W_{1}^{L} \circ \mathbb{F} L^{-1}\right)$, and the second CL system $\left(L_{2}, F_{2}^{L}, W_{2}^{L}\right)$ is transformed via $\mathbb{F} L_{2}$, to $\left(\tilde{H}_{2}, \tilde{B}_{2}=B_{\text {can }}, \tilde{F}_{2}^{H}=\right.$ $\left.F_{2}^{L} \circ \mathbb{F} L_{2}^{-1}, \tilde{W}_{2}^{H}=W_{2}^{L} \circ \mathbb{F} L_{2}^{-1}\right)$, where

$$
\begin{aligned}
& H_{1}(q, p)=\frac{1}{2}\left\langle p, m_{1}(q)^{-1} p\right\rangle+V_{1}(q), \\
& \tilde{H}_{2}(q, \tilde{p})=\frac{1}{2}\left\langle\tilde{p}, m_{2}(q)^{-1} \tilde{p}\right\rangle+V_{2}(q) .
\end{aligned}
$$

We now would like to show that checking the CL-equivalence of $\left(L_{1}, F_{1}^{L}, W_{1}^{L}\right)$ and $\left(L_{2}, F_{2}^{L}, W_{2}^{L}\right)$ is the same as checking the $\mathrm{CH}$-equivalence of their transformed $\mathrm{CH}$ systems. Thereby, we show that $\mathrm{CH}$-equivalence proves CL-equivalence. Since the two Legendre transformations in (45) and (46) are not the same in general, we need to pull-back the system $\left(\tilde{H}_{2}, \tilde{B}_{2}, \tilde{F}_{2}^{H}, \tilde{W}_{2}^{H}\right)$ via $\mathbb{F} L_{2} \circ \mathbb{F} L_{1}^{-1}=m_{2} \circ m_{1}^{-1} \in \Gamma\left(\operatorname{Aut}\left(T^{*} Q\right)\right)$, as in the commutative diagram in Figure 2.

Let $\left(H_{2}, B_{2}, F_{2}^{H}, W_{2}^{H}\right)=\left(\mathbb{F} L_{2} \circ \mathbb{F} L_{1}^{-1}\right)^{*}\left(\tilde{H}_{2}, \tilde{B}_{2}, \tilde{F}_{2}^{H}, \tilde{W}_{2}^{H}\right)$ where one computes

$$
\begin{aligned}
H_{2}(q, p) & =\frac{1}{2}\left\langle p, m_{1}(q)^{-1} m_{2}(q) m_{1}(q)^{-1} p\right\rangle+V_{2}(q) \\
B_{2}(q, p) & =\left[\begin{array}{cc}
O & \left(m_{1}(q) m_{2}(q)^{-1}\right)^{T} \\
-m_{1}(q) m_{2}(q)^{-1} & C_{m_{1} m_{2}^{-1}}(q, p)
\end{array}\right] \\
W_{2}^{H} & =\left(\mathbb{F} L_{2} \circ \mathbb{F} L_{1}^{-1}\right)^{*}\left(W_{2}^{L} \circ \mathbb{F} L_{2}^{-1}\right) \\
& =\left(\mathbb{F} L_{2} \circ \mathbb{F} L_{1}^{-1}\right)^{-1}\left(W_{2}^{L} \circ \mathbb{F} L_{2}^{-1}\right) \circ \mathbb{F} L_{2} \circ \mathbb{F} L_{1}^{-1} \\
& =\left(\mathbb{F} L_{1} \circ \mathbb{F} L_{2}^{-1}\right)\left(W_{2}^{L} \circ \mathbb{F} L_{1}^{-1}\right) .
\end{aligned}
$$


We will now show the following

$$
\left(H_{1}, B_{1}, F_{1}^{H}, W_{1}^{H}\right) \stackrel{H}{\sim}\left(H_{2}, B_{2}, F_{2}^{H}, W_{2}^{H}\right) \Longleftrightarrow\left(L_{1}, F_{1}^{L}, W_{1}^{L}\right) \stackrel{L}{\sim}\left(L_{2}, F_{2}^{L}, W_{2}^{L}\right) .
$$

First, HM-1 reads

$$
\begin{aligned}
W_{1}^{H}=W_{2}^{H} & \Longleftrightarrow W_{1}^{L} \circ \mathbb{F} L_{1}^{-1}=m_{1} m_{2}^{-1}\left(W_{2}^{L} \circ \mathbb{F} L_{1}^{-1}\right) \\
& \Longleftrightarrow W_{1}^{L}=m_{1} m_{2}^{-1} W_{2}^{L}
\end{aligned}
$$

whose right hand side is exactly ELM-1. Hence HM-1 for $\left(H_{1}, B_{1}, F_{1}^{H}, W_{1}^{H}\right)$ and $\left(H_{2}, B_{2}, F_{2}^{H}, W_{2}^{H}\right)$ is equivalent to ELM-1 for $\left(L_{1}, F_{1}^{L}, W_{1}^{L}\right)$ and $\left(L_{2}, F_{2}^{L}, W_{2}^{L}\right)$. Second, since $u_{1}^{H}, u_{2}^{H} \in W_{1}^{H}=W_{2}^{H}$, HM-2 can be equivalently written in coordinates as

$$
\left(\left[\begin{array}{c}
\dot{q} \\
\dot{p}
\end{array}\right]_{H_{1}}-\left[\begin{array}{c}
\dot{q} \\
\dot{p}
\end{array}\right]_{H_{2}}\right) \in \operatorname{vlift}\left(W_{1}^{H}(q)\right) \simeq O \oplus W_{1}^{H}(q)
$$

where

$$
\left[\begin{array}{c}
\dot{q} \\
\dot{p}
\end{array}\right]_{H_{i}}=X_{\left(H_{i}, B_{i}, F_{i}^{H}, u_{i}^{H}\right)}=B_{i}^{\sharp} \mathrm{d} H_{i}+\operatorname{vlift}\left(F_{i}^{H}\right)+\operatorname{vlift}\left(u_{i}^{H}\right)
$$

for each $i=1,2$ where the subscript $H_{i}$ denotes the $\mathrm{CH}$ system $\left(H_{i}, B_{i}, F_{i}^{H}, W_{i}^{H}\right)$ for simplicity.

Since $W_{1}^{H}=W_{2}^{H}=W_{1}^{L} \circ \mathbb{F} L_{1}^{-1}=m_{1} m_{2}^{-1} W_{2}^{L} \circ \mathbb{F} L_{2}^{-1}$ under HM-1, we can write the controls $u_{i}^{H} \in W_{1}^{H}$ as

$$
\begin{aligned}
& u_{1}^{H}=u_{1}^{L} \circ \mathbb{F} L_{1}^{-1} \\
& u_{2}^{H}=m_{1} m_{2}^{-1} u_{2}^{L} \circ \mathbb{F} L_{2}^{-1}
\end{aligned}
$$

for $u_{i}^{L} \in W_{i}^{L}$ which can be considered via the Legendre transformations $\mathbb{F} L_{1}$ and $\mathbb{F} L_{2}$ as the controls for $\left(L_{i}, F_{i}^{L}, W_{i}^{L}\right)$ for $i=1,2$, respectively.

Recall from (45) and (46) that $p=m_{1}(q) m_{2}(q)^{-1} \tilde{p}$. Using this, we find that

$$
\dot{q}_{H_{1}}-\dot{q}_{H_{2}}=m_{1}(q)^{-1} p-m_{2}(q)^{-1} \tilde{p}=m_{1}(q)^{-1} p-m_{2}(q)^{-1} m_{2}(q) m_{1}(q)^{-1} p=0 .
$$

The equation for $\dot{p}_{H_{1}}$ can be written in terms of $(q, \dot{q})$ as follows:

$$
\dot{p}_{H_{1}}=\frac{\mathrm{d}}{\mathrm{d} t} \frac{\partial L_{1}}{\partial \dot{q}}=\frac{\partial L_{1}}{\partial q}+F_{1}^{L}+u_{1}^{L} .
$$

The equation $\dot{p}_{\mathrm{H}_{2}}$ can be written in terms of $(q, \dot{q})$ as follows:

$$
\begin{aligned}
\dot{p}_{H_{2}} & =\frac{\mathrm{d}}{\mathrm{d} t}\left(m_{1} m_{2}^{-1} \tilde{p}\right) \\
& =\left(\mathbf{d}\left(m_{1} m_{2}^{-1}\right)[\dot{q}]\right) \tilde{p}+m_{1} m_{2}^{-1} \frac{\mathrm{d}}{\mathrm{d} t} \tilde{p}_{\tilde{H}_{2}} \\
& =\left(\mathbf{d}\left(m_{1} m_{2}^{-1}\right)[\dot{q}]\right) m_{2} \dot{q}+m_{1} m_{2}^{-1} \frac{\mathrm{d}}{\mathrm{d} t} \frac{\partial L_{2}}{\partial \dot{q}} \\
& =\left(\mathbf{d} m_{1}[\dot{q}]\right) \dot{q}-m_{1} m_{2}^{-1}\left(\mathbf{d} m_{2}[\dot{q}]\right) \dot{q}+m_{1} m_{2}^{-1}\left(\frac{\partial L_{2}}{\partial q}+F_{2}^{L}+u_{2}^{L}\right) \\
& =\left(\mathbf{d} m_{1}[\dot{q}]\right) \dot{q}-m_{1} m_{2}^{-1}\left(\left(\mathbf{d} m_{2}[\dot{q}]\right) \dot{q}-\frac{\partial L_{2}}{\partial q}-F_{2}^{L}-u_{2}^{L}\right) .
\end{aligned}
$$


Hence,

$$
\begin{aligned}
\dot{p}_{H_{2}}-\dot{p}_{H_{1}} & =\left(\left(\mathbf{d} m_{1}[\dot{q}]\right) \dot{q}-\frac{\partial L_{1}}{\partial q}-F_{1}^{L}-u_{1}^{L}\right)-m_{1} m_{2}^{-1}\left(\left(\mathbf{d} m_{2}[\dot{q}]\right) \dot{q}-\frac{\partial L_{2}}{\partial q}-F_{2}^{L}-u_{2}^{L}\right) \\
& =\left(m_{1} \ddot{q}+\left(\mathbf{d} m_{1}[\dot{q}]\right) \dot{q}-\frac{\partial L_{1}}{\partial q}-F_{1}^{L}-u_{1}^{L}\right)-m_{1} m_{2}^{-1}\left(m_{2} \ddot{q}+\left(\mathbf{d} m_{2}[\dot{q}]\right) \dot{q}-\frac{\partial L_{2}}{\partial q}-F_{2}^{L}-u_{2}^{L}\right) \\
& =\left(\mathcal{E} \mathcal{L}\left(L_{1}\right)-F_{1}^{L}\right)-m_{1} m_{2}^{-1}\left(\mathcal{E L}\left(L_{2}\right)-F_{2}^{L}\right)-u_{1}^{L}+m_{1} m_{2}^{-1} u_{2}^{L}
\end{aligned}
$$

Therefore (48) holds if and only if

$$
\operatorname{Im}\left[\left(\mathcal{E} \mathcal{L}\left(L_{1}\right)-F_{1}^{L}\right)-m_{1} m_{2}^{-1}\left(\mathcal{E} \mathcal{L}\left(L_{2}\right)-F_{2}^{L}\right)\right] \subset W_{2}^{L}
$$

since $u_{1}^{L}, m_{1} m_{2}^{-1} u_{2}^{L} \in W_{1}^{L}=m_{1} m_{2}^{-1} W_{2}^{L}$. Therefore, we have shown (47). Finally, one can easily show from (48) and (49) that condition (17) on the inputs $u_{i}^{L}$ 's is equivalent to condition (28) on the inputs $u_{i}^{H}$ 's.

We make a remark on an alternative way of comparison of HM-2 and ELM-2. One can show by a brute-force coordinate computation that

$$
\begin{aligned}
{\left[\left(B_{2}^{\sharp} \mathbf{d} H_{2}+\operatorname{vlift}\left(F_{2}^{H}\right)\right)-\left(B_{1}^{\sharp} \mathbf{d} H_{1}+\operatorname{vlift}\left(F_{1}^{H}\right)\right)\right] } \\
\quad \simeq O \oplus\left[\left(\mathcal{E} \mathcal{L}\left(L_{1}\right)-F_{1}^{L}\right)-m_{1} m_{2}^{-1}\left(\mathcal{E} \mathcal{L}\left(L_{2}\right)-F_{2}^{L}\right)\right] .
\end{aligned}
$$

This computation is much more complicated and it does not directly lead to the equivalence of (17) and (28). This is why we did not choose this brute-force computational method in this paper.

CL-Equivalence Proves CH-Equivalence. We now show that the Hamiltonian matching conditions of simple $\mathrm{CH}$ systems can be derived from those of simple CL systems. Consider two simple $\mathrm{CH}$ systems $\left(H_{1}, B_{1}, F_{1}^{H}, W_{1}^{H}\right)$ and $\left(H_{2}, B_{2}, F_{2}^{H}, W_{2}^{H}\right)$ with $H_{i}(q, p)=\frac{1}{2}\left\langle p, m_{i}^{-1}(q) p\right\rangle+V_{i}(q)$ for $i=1,2$. By Proposition 3.6 and Proposition 3.9, without loss of generality, we may assume that

$$
B_{1}=B_{\mathrm{can}} ; \quad \psi_{B_{2}}^{*} B_{2}=B_{\mathrm{can}}
$$

In coordinates, we write $B_{2}$ and $\psi_{B_{2}}$ as follows:

$$
B_{2}(q, p)=\left[\begin{array}{cc}
O & K_{2}(q)^{T} \\
-K_{2}(q) & C_{K_{2}}(q, p)
\end{array}\right]
$$

and

$$
(q, p)=\psi_{B_{2}}(q, \tilde{p})=\left(q, K_{2}(q) \tilde{p}\right)
$$

Consider the pull-back system $\left(\tilde{H}_{2}, \tilde{B}_{2}=B_{\text {can }}, \tilde{F}_{2}^{H}, \tilde{W}_{2}^{H}\right):=\left(\psi_{B_{2}}\right)^{*}\left(H_{2}, B_{2}, F_{2}^{H}, W_{2}^{H}\right)$ with

$$
H_{2}(q, \tilde{p})=\frac{1}{2}\left\langle\tilde{p}, \tilde{m}_{2}^{-1}(q) \tilde{p}\right\rangle+V_{2}(q) ; \quad \tilde{m}_{2}:=\psi_{B_{2}}^{-1} m_{2}\left(\psi_{B_{2}}^{*}\right)^{-1}
$$

where $\psi_{B_{2}}^{*} \in \Gamma(\operatorname{Aut}(T Q))$ is the dual of $\psi_{B_{2}} \in \Gamma\left(\operatorname{Aut}\left(T^{*} Q\right)\right)$.

The system $\left(H_{1}, B_{1}, F_{1}^{H}, W_{1}^{H}\right)$ is transformed via the inverse Legendre transformation $(q, \dot{q})=\mathbb{F} H_{1}(q, p)$ $=\left(q, m_{1}(q)^{-1} p\right)$ to the CL system $\left(L_{1}, F_{1}^{L}, W_{1}^{L}\right)$, where

$$
L_{1}(q, \dot{q})=\frac{1}{2}\left\langle\dot{q}, m_{1} \dot{q}\right\rangle-V_{1}(q), \quad F_{1}^{L}=F_{1}^{H} \circ \mathbb{F} H_{1}^{-1} \quad \text { and } \quad W_{1}^{L}=W_{1}^{H} \circ \mathbb{F} H_{1}^{-1} .
$$




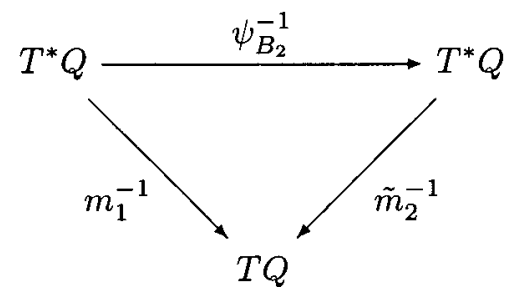

FiguRE 3. Diagram of inverse Legendre transformations.

The system $\left(\tilde{H}_{2}, \tilde{B}_{2}, \tilde{F}_{2}^{H}, \tilde{W}_{2}^{H}\right)$ is transformed via the inverse Legendre transformation $(q, \dot{q})=\mathbb{F} \tilde{H}_{2}(q, \tilde{p})=$ $\left(q, \tilde{m}_{2}^{-1}(q) \tilde{p}\right)$ to the CL system $\left(L_{2}, F_{2}^{L}, W_{2}^{L}\right)$, where

$$
L_{2}(q, \dot{q})=\frac{1}{2}\left\langle\dot{q}, \tilde{m}_{2} \dot{q}\right\rangle-V_{2}(q), \quad F_{2}^{L}=\tilde{F}_{2}^{H} \circ \mathbb{F} \tilde{H}_{2}^{-1} \quad \text { and } \quad W_{2}^{L}=\tilde{W}_{2}^{H} \circ \mathbb{F} \tilde{H}_{2}^{-1} .
$$

The diagram in Figure 3 commutes if and only if $\psi_{B_{2}}=m_{1} \tilde{m}_{2}^{-1}$, which by the definition of $\tilde{m}_{2}$ in (50) is equivalent to

$$
\psi_{B_{2}}=m_{2} m_{1}^{-1}
$$

i.e., in a matrix form $K_{2}(q)=m_{2}(q) m_{1}(q)^{-1}$. We will show that

$$
\left(H_{1}, B_{1}, F_{1}^{H}, W_{1}^{H}\right) \stackrel{H}{\sim}\left(H_{2}, B_{2}, F_{2}^{H}, W_{2}^{H}\right) \Longleftrightarrow\left[\left(L_{1}, F_{1}^{L}, W_{1}^{L}\right) \stackrel{L}{\sim}\left(L_{2}, F_{2}^{L}, W_{2}^{L}\right)\right]+(51) .
$$

Under (51), one computes

$$
\begin{aligned}
m_{1} \tilde{m}_{2}^{-1} W_{2}^{L} & =\psi_{B}\left(\psi_{B_{2}}^{*} W_{2}^{H}\right) \circ \mathbb{F} L_{2} \\
& =\psi_{B}\left(\psi_{B_{2}}^{-1} W_{2}^{H} \circ \psi_{B_{2}}\right) \circ \mathbb{F} L_{2} \\
& =W_{2}^{H} \circ m_{1}=W_{2}^{H} \circ \mathbb{F} L_{1} .
\end{aligned}
$$

First, under (51), ELM-1 reads

$$
W_{1}^{L}=m_{1} \tilde{m}_{2}^{-1} W_{2}^{L} \Longleftrightarrow W_{1}^{H}=W_{2}^{H}
$$

which is HM-1 for $\left(H_{1}, B_{1}, F_{1}, W_{1}\right)$ and $\left(H_{2}, B_{2}, F_{2}, W_{2}\right)$. Second, since $u_{1}^{H}, u_{2}^{H} \in W_{1}^{H}=W_{2}^{H}$, HM-2 can be equivalently written in coordinates as (48). One can compute

$$
\dot{q}_{H_{1}}-\dot{q}_{H_{2}}=m_{1}(q)^{-1} p-K_{2}(q)^{T} m_{2}(q)^{-1} p .
$$

Hence, the first half of $\mathbf{H M - 2}$ reads

$$
\dot{q}_{H_{1}}-\dot{q}_{H_{2}}=0 \Longleftrightarrow K_{2}(q)=m_{2}(q) m_{1}(q)^{-1}
$$

which is the commutativity condition (51). The remaining half of HM-2 reads $\dot{p}_{H_{1}}-\dot{p}_{H_{2}} \in W_{2}^{H}$. By a similar computation carried out in the paragraph entitled CH-equivalence Proves CL-Equivalence, one can show that

$$
\dot{p}_{H_{1}}-\dot{p}_{H_{2}} \in W_{2}^{H} \Longleftrightarrow \text { ELM-2 }
$$

under (51). One can also readily show that condition (17) on the inputs $u_{i}^{L}$ 's is equivalent to the condition (28) on the inputs $u_{i}^{H}$ 's. Therefore we have established (52). 
The Main Theorem. We summarize the preceding discussion in the following theorem ${ }^{6}$.

Theorem 4.1. The method of controlled Lagrangians is equivalent to that of controlled Hamiltonians for simple mechanical systems. Namely, the following holds:

1: Let $\left(L_{i}, F_{i}^{L}, W_{i}^{L}\right), i=1,2$, be two simple $C L$ systems, and let $\left(H_{i}, B_{\mathrm{can}}, F_{i}^{H}, W_{i}^{H}\right)$ be the associated simple $C H$ systems Legendre-transformed via $\mathbb{F} L_{i}$ from the $C L$ system $\left(L_{i}, F_{i}^{L}, W_{i}^{L}\right)$ for $i=1,2$, respectively. Then,

$$
\left(L_{1}, F_{1}^{L}, W_{1}^{L}\right) \stackrel{L}{\sim}\left(L_{2}, F_{2}^{L}, W_{2}^{L}\right) \Longleftrightarrow\left(H_{1}, B_{\mathrm{can}}, F_{1}^{H}, W_{1}^{H}\right) \stackrel{H}{\sim}\left(\mathbb{F} L_{2} \circ \mathbb{F} L_{1}^{-1}\right)^{*}\left(H_{2}, B_{\mathrm{can}}, F_{2}^{H}, W_{2}^{H}\right),
$$

where $B_{\text {can }}$ is the canonical Poisson tensor on $T^{*} Q$.

2: Let $\left(H_{i}, B_{i}, F_{i}^{H}, W_{i}^{H}\right), i=1,2$, be two simple $C H$ systems. Decompose $B_{i}$ into its regular part $B_{\mathrm{r}, i}$ and its gyroscopic part $B_{\mathrm{gr}, i}$ so that

$$
\left(H_{i}, B_{i}, F_{i}^{H}, W_{i}^{H}\right) \stackrel{H}{\sim}\left(H_{i}, B_{\mathrm{r}, i}, F_{i}^{H}+F_{\mathrm{gr}, i}^{H}, W_{i}^{H}\right)
$$

where the gyroscopic force $F_{\mathrm{gr}, i}^{H}$ is defined by $\operatorname{vlift}\left(F_{\mathrm{gr}, i}\right)=B_{\mathrm{gr}, i}^{\sharp} \mathbf{d} H_{i}$ (see Prop. 3.6). Then there exist $\psi_{1}, \psi_{2} \in \Gamma\left(\operatorname{Aut}\left(T^{*} Q\right)\right)$ and two simple $C H$ systems $\left(\hat{H}_{i}, B_{\mathrm{can}}, \hat{F}_{i}^{H}, \hat{W}_{i}^{H}\right), i=1,2$ such that

$$
\psi_{i}^{*}\left(H_{i}, B_{\mathrm{r}, i}, F_{i}^{H}+F_{\mathrm{gr}, i}^{H}, W_{i}^{H}\right)=\left(\hat{H}_{i}, B_{\mathrm{can}}, \hat{F}_{i}^{H}, \hat{W}_{i}^{H}\right) \quad i=1,2,
$$

and finally

$$
\left(H_{1}, B_{1}, F_{1}^{H}, W_{1}^{H}\right) \stackrel{H}{\sim}\left(H_{2}, B_{2}, F_{2}^{H}, W_{2}^{H}\right) \Longleftrightarrow\left(L_{1}, F_{1}^{L}, W_{1}^{L}\right) \stackrel{L}{\sim}\left(L_{2}, F_{2}^{L}, W_{2}^{L}\right)
$$

where $\left(L_{i}, F_{i}^{L}, W_{i}^{L}\right)$ is the simple $C L$ system inverse-Legendre-transformed via $\mathbb{F} \hat{H}_{i}$ from the CH system $\left(\hat{H}_{i}, B_{\text {can }}, \hat{F}_{i}^{H}, \hat{W}_{i}^{H}\right)$ for $i=1,2$, respectively.

Here is another way to state this result which is shorter and perhaps easier to digest, but gives a little less information.

Corollary 4.2. The method of controlled Lagrangians is equivalent to that of controlled Hamiltonians for simple mechanical systems in the following sense. For any two simple $C L$ systems $\left(L_{i}, F_{i}^{L}, W_{i}^{L}\right), i=1,2$, there exist two associated simple CH systems $\left(H_{i}, B_{i}, F_{i}^{H}, W_{i}^{H}\right), i=1,2$, such that

$$
\left(L_{1}, F_{1}^{L}, W_{1}^{L}\right) \stackrel{L}{\sim}\left(L_{2}, F_{2}^{L}, W_{2}^{L}\right) \Longleftrightarrow\left(H_{1}, B_{1}, F_{1}^{H}, W_{1}^{H}\right) \stackrel{H}{\sim}\left(H_{2}, B_{2}, F_{2}^{H}, W_{2}^{H}\right),
$$

and vice-versa.

\section{The method of Collocated/NON-COllocated PARTial FEEDBACK Linearization}

Even though the controlled Lagrangian method was originally developed for stabilization of equilibria using energy shaping and dissipation, here we show that one can put the collocated/non-collocated partial feedback linearization property of underactuated mechanical systems into the controlled Lagrangian framework (see Spong's work [31] and references therein for more about the collocated/non-collocated partial feedback linearization).

\footnotetext{
${ }^{6}$ The notation and terminology used in this theorem can be found in the body of the paper as follows. Definition 2.3 and Definition 3.2 give the definitions of CL-equivalence, $\stackrel{L}{\sim}$, and the CH-equivalence relation, $\stackrel{H}{\sim}$. Definition 2.2 and Definition 3.5 give the definitions of simple CL systems and simple CH systems. The beginning of Section 4 provides the definition of the Legendre transformation and the construction of $\psi_{1}$ and $\psi_{2}$. Proposition 3.9 and the remarks before it discuss the definition of pull-back systems.
} 
For simplicity, take $Q=\mathbb{R}^{n_{1}} \times \mathbb{R}^{n_{2}}$ to be the configuration space and use $q=\left(q_{1}, q_{2}\right) \in \mathbb{R}^{n_{1}} \times \mathbb{R}^{n_{2}}$ as coordinates. Let $(L, F=0, W)$ be a given CL system (with no external forces) and with $W(q)=0 \times \mathbb{R}^{n_{2}}$ for all $q \in Q$ and

$$
L(q, \dot{q})=\frac{1}{2}\left[\begin{array}{l}
\dot{q}_{1} \\
\dot{q}_{2}
\end{array}\right]^{T}\left[\begin{array}{ll}
m_{11}(q) & m_{12}(q) \\
m_{21}(q) & m_{22}(q)
\end{array}\right]\left[\begin{array}{l}
\dot{q}_{1} \\
\dot{q}_{2}
\end{array}\right]-V(q)
$$

Then the Euler-Lagrange equation of this system can be written in the following form (as in [31]):

$$
\begin{aligned}
& m_{11} \ddot{q}_{1}+m_{12} \ddot{q}_{2}+h_{1}(q, \dot{q})+\phi_{1}(q)=0, \\
& m_{21} \ddot{q}_{1}+m_{22} \ddot{q}_{2}+h_{2}(q, \dot{q})+\phi_{2}(q)=\tau
\end{aligned}
$$

with control $\tau: T Q \rightarrow W$ where $h_{i}$ includes all $\dot{q}$-dependent terms and $\phi_{i}$ contains the terms from the potential energy.

Collocated Linearization. Define the new CL system $\left(L_{c}, F_{c}, W_{c}\right)$ as follows:

$$
\begin{aligned}
L_{c}(q, \dot{q}) & =\frac{1}{2} \dot{q}_{1}^{T} m_{11} \dot{q}_{1}+\frac{1}{2} \dot{q}_{2}^{T} \dot{q}_{2} \\
F_{c}(q, \dot{q}) & =\left[\begin{array}{c}
-h_{1}-\phi_{1}+\left(\mathbf{d} m_{11}[\dot{q}]\right) \dot{q}_{1}-\frac{\partial L_{c}}{\partial q_{1}} \\
-\frac{\partial L_{c}}{\partial q_{2}}
\end{array}\right] \\
W_{c} & =\left\langle\mathbf{d} q_{2}^{i}-\left(m_{12}\right)_{\alpha i} \mathbf{d} q_{1}^{\alpha} \mid i=1, \ldots, n_{2}\right\rangle \\
& =\text { the subbundle spanned by the columns of }\left[\begin{array}{c}
-m_{12}(q) \\
I_{n_{2}}
\end{array}\right]
\end{aligned}
$$

where $I_{n_{2}}$ is the $n_{2} \times n_{2}$ identity matrix. If a part of $F_{c}$ is a potential force, i.e., the differential of a function, one can combine it into the Lagrangian $L_{c}$ as a potential function. A control to the system $\left(L_{c}, F_{c}, W_{c}\right)$ can be written via a map $u_{c}: T Q \rightarrow \mathbb{R}^{n_{2}}$ as follows:

$$
\left[\begin{array}{c}
-m_{12}(q) \\
I_{n_{2}}
\end{array}\right] u_{c}(q, \dot{q}) \in W_{c}(q)
$$

One can check that the given system $(L, 0, W)$ is CL-equivalent to the system $\left(L_{c}, F_{c}, W_{c}\right)$ and the EulerLagrange equation of $\left(L_{c}, F_{c}, W_{c}\right)$ is given by

$$
\begin{aligned}
m_{11} \ddot{q}_{1}+h_{1}+\phi_{1} & =-m_{12} u_{c} \\
\ddot{q}_{2} & =u_{c}
\end{aligned}
$$

with $u_{c}: T Q \rightarrow \mathbb{R}^{n_{2}}$. If we write the control for $\left(L_{c}, F_{c}, W_{c}\right)$ in the form (53), then equation (17) is written as

$$
\tau=h_{2}+\phi_{2}-m_{21} m_{11}^{-1}\left(h_{1}+\phi_{1}\right)+\left(m_{22}-m_{21} m_{11}^{-1} m_{12}\right) u_{c}
$$

so that the closed-loop system $(L, 0, \tau)$ and $\left(L_{c}, F_{c}, u_{c}\right)$ produce the same equations of motion. This coincides with the notion of collocated partial feedback linearization in [31].

As already indicated at the end of Section 2, if the control bundle is integrable, it allows us to find a set of local coordinates for the configuration space $Q$ which is convenient for doing stability analysis (as was the case in [3]). The integrability condition of the control bundle $W_{c}$ is given by (the curvature condition)

$$
\frac{\partial A_{\alpha}^{i}}{\partial q_{2}^{j}} A_{\beta}^{j}-\frac{\partial A_{\beta}^{i}}{\partial q_{2}^{j}} A_{\alpha}^{j}+\frac{\partial A_{\alpha}^{i}}{\partial q_{1}^{\beta}}-\frac{\partial A_{\beta}^{i}}{\partial q_{1}^{\alpha}}=0
$$


for $1 \leq \alpha, \beta \leq n_{1}$ and $1 \leq i, j \leq n_{2}$ where $A_{\alpha}^{i}$ is the $(i, \alpha)$-th element of the matrix $m_{21}=m_{12}^{T}$ and $\left(q_{1}, q_{2}\right)$ $=\left(q_{1}^{\alpha}, q_{2}^{i}\right)$; this is seen from the fact that $W_{c}$ is spanned by the set $\left\{d q_{2}^{i}-A_{\alpha}^{i} d q_{1}^{\alpha} \mid 1 \leq \alpha \leq n_{1}, 1 \leq i \leq n_{2}\right\}$.

Non-Collocated Linearization. For non-collocated linearization [31] makes the assumption that the submatrix $m_{12}(q)$ is onto, i.e., $\operatorname{rank}\left(m_{12}(q)\right)=l$ for all $q \in Q$. Then, there is a pseudo-inverse $m_{12}^{\dagger}:=m_{12}^{T}\left(m_{12} m_{12}^{T}\right)^{-1}$ such that $m_{12} m_{12}^{\dagger}=I_{n_{1}}$ with $I_{n_{1}}$ the $n_{1} \times n_{1}$ identity matrix. For this assumption to hold, it is necessary that $n_{2} \geq n_{1}$, i.e., the number of actuation degrees of freedom should be at least as big as the number of unactuated degrees of freedom. The same property was used in [3].

Define the system $\left(L_{n}, F_{n}, W_{n}\right)$ as follows:

$$
\begin{aligned}
L_{n}(q, \dot{q}) & =\frac{1}{2} \dot{q}_{1}^{T} \dot{q}_{1}+\frac{1}{2} \dot{q}_{2}^{T} \dot{q}_{2}, \\
F_{n}(q, \dot{q}) & =\left[\begin{array}{c}
0 \\
-m_{12}(q)^{\dagger}\left(h_{1}(q, \dot{q})+\phi_{1}(q)\right)
\end{array}\right] \\
W_{n} & =\left\langle\mathbf{d} q_{1}^{i}-\left(m_{12}^{\dagger} m_{11}\right)_{\alpha i} \mathbf{d} q_{2}^{\alpha} \mid i=1, \ldots, n_{1}\right\rangle \\
& =\text { subbundle spanned by the columns of }\left[\begin{array}{c}
I_{n_{1}} \\
-m_{12}(q)^{\dagger} m_{11}(q)
\end{array}\right]
\end{aligned}
$$

where, as above, one can move any potential force parts of $F_{n}$ into the Lagrangian $L_{n}$ as a potential function. A control for the system $\left(L_{n}, F_{n}, W_{n}\right)$ can be written via a map $u_{n}: T Q \rightarrow \mathbb{R}^{n_{1}}$ as follows:

$$
\left[\begin{array}{c}
I_{n_{1}} \\
-m_{12}(q)^{\dagger} m_{11}(q)
\end{array}\right] u_{n}(q, \dot{q}) \in W_{n}(q) .
$$

Notice that $\operatorname{dim} W_{n}(q) \leq \operatorname{dim} W(q)$. Hence, it is appropriate to use the concept of CL-inclusion rather than CL-equivalence. Indeed, one can easily check that the given system $(L, 0, W)$ includes the system $\left(L_{n}, F_{n}, W_{n}\right)$ and the Euler-Lagrange equations of $\left(L_{n}, F_{n}, W_{n}\right)$ are written as

$$
\begin{aligned}
& \ddot{q}_{1}=u_{n} \\
& \ddot{q}_{2}=-m_{12}^{\dagger} m_{11}\left(u_{n}+h_{1}+\phi_{1}\right)
\end{aligned}
$$

with $u_{n}: T Q \rightarrow \mathbb{R}^{n_{2}}$. If we write the control for $\left(L_{n}, F_{n}, W_{n}\right)$ in the form (56), then condition (17) is written as

$$
\tau=h_{2}+\phi_{2}-m_{22} m_{12}^{\dagger}\left(h_{1}+\phi_{1}\right)+\left(m_{21}-m_{22} m_{12}^{\dagger} m_{11}\right) u_{n}
$$

such that the two closed-loop systems $(L, 0, \tau)$ and $\left(L_{n}, F_{n}, u_{n}\right)$ produce the same equations of motion. This coincides with the non-collocated partial feedback linearization in [31].

\section{Future Directions.}

1. It reasonable to expect that one can apply the same techniques to nonholonomic systems, following the work of $[14]$ and $[42,43]$.

2. It would be interesting to consider the case of degenerate almost Poisson structures. This would take one into the realm of Dirac structures; see $[5,15,17,20,27]$.

3. Reduction theory for CL and CH systems with symmetry will be the subject of a companion paper.

We are grateful to the authors of [29] and of [2] for providing us advance copies of their papers and for helpful correspondence. 


\section{REFERENCES}

[1] D. Auckly, L. Kapitanski and W. White, Control of nonlinear underactuated systems. Comm. Pure Appl. Math. 53 (2000) 354-369. (See related papers at http://www.math.ksu.edu/d̃av/).

[2] G. Blankenstein, R. Ortega and A. van Der Schaft, The matching conditions of controlled Lagrangians and IDA passivity based control. Preprint (2001).

[3] A.M. Bloch, D.E. Chang, N.E. Leonard and J.E. Marsden, Controlled Lagrangians and the stabilization of mechanical systems II: Potential shaping. IEEE Trans. Automat. Control 46 (2001) 1556-1571.

[4] A.M. Bloch, D.E. Chang, N.E. Leonard, J.E. Marsden and C.A. Woolsey, Stabilization of Mechanical Systems with StructureModifying Feedback. Presented at the 2001 SIAM Conf. on Control and its Applications, http://www. aoe.vt.edu/ cwoolsey/Lectures/SIAM.7.01.html

[5] A.M. Bloch and P.E. Crouch, Representation of Dirac structures on vector space and nonlinear L-C circuits, in Proc. Symp. on Appl. Math., AMS 66 (1998) 103-118.

[6] A.M. Bloch and P.E. Crouch, Optimal control, optimization and analytical mechanics, in Mathematical Control Theory, edited by J. Baillieul and J. Willems Springer (1998) 268-321.

[7] A.M. Bloch, P.S. Krishnaprasad, J.E. Marsden and G. Sánchez De Alvarez, Stabilization of rigid body dynamics by internal and external torques. Automatica 28 (1992) 745-756.

[8] A.M. Bloch, N.E. Leonard and J.E. Marsden, Stabilization of mechanical systems using controlled Lagrangians, in Proc. IEEE CDC 36 (1997) 2356-2361.

[9] A.M. Bloch, N.E. Leonard and J.E. Marsden, Controlled Lagrangians and the stabilization of mechanical systems I: The first matching theorem. IEEE Trans. Automat. Control 45 (2000) 2253-2270.

[10] A.M. Bloch, N.E. Leonard and J.E. Marsden, Controlled Lagrangians and the stabilization of Euler-Poincaré mechanical systems. Int. J. Robust Nonlinear Control 11 (2001) 191-214.

[11] R.W. Brockett, Control theory and analytical mechanics, in 1976 Ames Research Center (NASA) Conference on Geometric Control Theory, edited by R. Hermann and C. Martin. Math Sci Press, Brookline, Massachusetts, Lie Groups: History, Frontiers, and Applications VII (1976) 1-46.

[12] A. Cannas Da Silva and A. Weinstein, Geometric Models for Noncommutative Algebras. Amer. Math. Soc., Berkeley Mathematics Lecture Notes (1999).

[13] H. Cendra, J.E. Marsden and T.S. Ratiu, Lagrangian Reduction by Stages. Memoirs of the Amer. Math. Soc. 152 (2001).

[14] H. Cendra, J.E. Marsden and T.S. Ratiu, Geometric mechanics, Lagrangian reduction and nonholonomic systems, in Mathematics Unlimited-2001 and Beyond, edited by B. Enquist and W. Schmid. Springer-Verlag, New York (2001) 221-273.

[15] T. Courant, Dirac manifolds. Trans. Amer. Math. Soc. 319 (1990) 631-661.

[16] P.E. Crouch and A. J. van der Schaft, Variational and Hamiltonian Control Systems. Springer-Verlag, Berlin, Lecture Notes in Control and Inform. Sci. 101 (1987).

[17] I. Dorfman,Dirac Structures and Integrability of Nonlinear Evolution Equations. Chichester: John Wiley (1993).

[18] J. Hamberg, General matching conditions in the theory of controlled Lagrangians, in Proc. IEEE CDC (1999) $2519-2523$.

[19] J. Hamberg, Controlled Lagrangians, symmetries and conditions for strong matching, in Lagrangian and Hamiltonian Methods for Nonlinear Control: A Proc. Volume from the IFAC Workshop, edited by N.E. Leonard and R. Ortega. Pergamon (2000) $57-62$.

[20] A. Ibort, M. De Leon, J.C. Marrero and D. Martin De Diego, Dirac brackets in constrained dynamics. Fortschr. Phys. 30 (1999) 459-492.

[21] S.M. Jalnapurkar and J.E. Marsden, Stabilization of relative equilibria II. Regul. Chaotic Dyn. 3 (1999) 161-179.

[22] S.M. Jalnapurkar and J.E. Marsden, Stabilization of relative equilibria. IEEE Trans. Automat. Control 45 (2000) 1483-1491.

[23] H.K. Khalil, Nonlinear Systems. Prentice-Hall, Inc. Second Edition (1996).

[24] W.S. Koon and J.E. Marsden, The Poisson reduction of nonholonomic mechanical systems. Reports on Math. Phys. 42 (1998) 101-134.

[25] P.S. Krishnaprasad, Lie-Poisson structures, dual-spin spacecraft and asymptotic stability. Nonl. Anal. Th. Meth. and Appl. 9 (1985) 1011-1035.

[26] J.E. Marsden and T.S. Ratiu, Introduction to Mechanics and Symmetry. Springer-Verlag, Texts in Appl. Math. 17 (1999) Second Edition.

[27] B.M. Maschke, A.J. van der Schaft and P.C. Breedveld, An Intrinsic Hamiltonian Formulation of the Dynamics of LC-Circuits. IEEE Trans. Circuits and Systems 42 (1995) 73-82.

[28] R. Ortega, A. Loria, P.J. Nicklasson and H. Sira-Ramirez, Passivity-based Control of Euler-Lagrange Systems. SpringerVerlag. Communication \& Control Engineering Series (1998).

[29] R. Ortega, M.W. Spong, F. Gómez-Estern and G. Blankenstein, Stabilization of underactuated mechanical systems via interconnection and damping assignment. IEEE Trans. Aut. Control (to appear).

[30] G. Sánchez De Alvarez, Controllability of Poisson control systems with symmetry. Amer. Math. Soc., Providence, RI., Contemp. Math. 97 (1989) 399-412. 
[31] M.W. Spong, Underactuated mechanical systems, in Control Problems in Robotics and Automation, edited by B. Siciliano and K.P. Valavanis. Spinger-Verlag, Lecture Notes in Control and Inform. Sci. 230. [Presented at the International Workshop on Control Problems in Robotics and Automation: Future Directions Hyatt Regency, San Diego, California (1997).]

[32] A.J. van der Schaft, Hamiltonian dynamics with external forces and observations. Math. Syst. Theory 15 (1982) $145-168$.

[33] A.J. van der Schaft, System Theoretic Descriptions of Physical Systems, Doct. Dissertation, University of Groningen; also CWI Tract \#3, CWI, Amsterdam (1983).

[34] A.J. van der Schaft, Stabilization of Hamiltonian systems. Nonlinear Anal. Theor. Meth. Appl. 10 (1986) 1021-1035.

[35] A.J. van der Schaft, $L_{2}$-Gain and Passivity Techniques in Nonlinear Control. Springer-Verlag, Commun. Control Engrg. Ser. (2000).

[36] A.J. van der Schaft and B. Maschke, On the Hamiltonian formulation of nonholonomic mechanical systems. Rep. Math. Phys. 34 (1994) 225-233.

[37] J.C. Willems, System theoretic models for the analysis of physical systems. Ricerche di Automatica 10 (1979) 71-106.

[38] C.A. Woolsey, Energy Shaping and Dissipation: Underwater Vehicle Stabilization Using Internal Rotors, Ph.D. Thesis. Princeton University (2001).

[39] C.A. Woolsey, A.M. Bloch, N.E. Leonard and J.E. Marsden, Physical dissipation and the method of controlled Lagrangians, in Proc. of the European Control Conference (2001) 2570-2575.

[40] C.A. Woolsey, A.M. Bloch, N.E. Leonard and J.E. Marsden, Dissipation and controlled Euler-Poincaré systems, in Proc. IEEE CDC (2001) 3378-3383.

[41] C.A. Woolsey and N.E. Leonard, Modification of Hamiltonian structure to stabilize an underwater vehicle, in Lagrangian and Hamiltonian Methods for Nonlinear Control: A Proc. Volume from the IFAC Workshop edited by N.E. Leonard and R. Ortega. Pergamon (2000) 175-176.

[42] D.V. Zenkov, A.M. Bloch, N.E. Leonard and J.E. Marsden, Matching and stabilization of the unicycle with rider, Lagrangian and Hamiltonian Methods for Nonlinear Control: A Proc. Volume from the IFAC Workshop, edited by N.E. Leonard and R. Ortega. Pergamon (2000) 177-178.

[43] D.V. Zenkov, A.M. Bloch and J.E. Marsden, Flat nonholonomic matching, Proc ACC 2002 (to appear). 